\title{
Regularity and Coexistence Problems for Strongly Coupled Elliptic Systems
}

\author{
Dung Le, Linh V. NGUyen \& TOAN T. NGUyen
}

\begin{abstract}
Boundedness and Hölder regularity of solutions to a class of strongly coupled elliptic systems are investigated. The Hölder estimates for the gradients of solutions are also established. Finally, the fixed point theory is applied to prove existence of positive solution(s) for general cross diffusion elliptic systems.
\end{abstract}

\section{INTRODUCTION}

In this paper, we study the boundedness and regularity of weak solutions and the coexistence problems of the following strongly coupled elliptic system:

$$
\left\{\begin{array}{l}
-\operatorname{div}\left[P^{u}(u, v) \nabla u+P^{v}(u, v) \nabla v\right]=f(u, v), \\
-\operatorname{div}\left[Q^{u}(u, v) \nabla u+Q^{v}(u, v) \nabla v\right]=g(u, v),
\end{array}\right.
$$

on a bounded domain $\Omega$ of $\mathbb{R}^{n}$ (for any $n \geq 1$ ) with smooth boundary $\partial \Omega$.

The system above arises in many important applications. For instance, it can be used to describe cross diffusion systems modeling the random movements of species under investigation, their interaction with each other as well as with the culture they live in. Here, the functions $f, g$ are the so-called reaction terms that model the interaction among the species. In the higher order part, $P^{u}, Q^{v}$ are the self diffusion rates, while $P^{v}, Q^{u}$ are the cross diffusion rates describing the gradient effect of the species on the movement of the other. In particular, $P^{v}<0$ implies that the species $u$ is moving toward the high density concentration region of $v$, whereas one may also consider the case $P^{v}>0$ which describes the movement of $u$ in the opposite direction. The introduction of cross diffusion terms $P^{v}, Q^{u}$ into classical reaction diffusion systems allows the mathematical models to capture much more realistic features of important phenomena in physics, biology, ecology, and engineering sciences. Obviously, this strong coupling causes 
enormous difficulties in the analytical treatment. Many fundamental questions are left open; and techniques which worked successfully for reaction-diffusion (weakly coupled) systems are no longer applicable.

In applications, we also have to specify the behavior of solutions to (1.1) on the smooth boundary $\partial \Omega$. Most prominently, one can consider the 'influx' Robin boundary conditions

$$
\frac{\partial u}{\partial v}+r_{1}(x) u=\frac{\partial v}{\partial v}+r_{2}(x) v=0, \quad \text { on } \partial \Omega
$$

where $r_{1}(x), r_{2}(x) \geq 0$ are two bounded functions given on $\partial \Omega$. No-flux conditions, or Neumann type when $r_{1} \equiv r_{2} \equiv 0$, are also of much interest. Prescribed value conditions, or Dirichlet type, are also investigated, that is,

$$
u(x)=u_{0}(x) \quad \text { and } \quad v(x)=v_{0}(x), \quad \text { for } x \in \partial \Omega,
$$

where $u_{0}, v_{0}$ are smooth bounded functions given on $\partial \Omega$.

In fact, we can as well consider mixed boundary conditions, where the Robin or Neumann conditions are given on $\partial \Omega_{1}$, a union of components $\partial \Omega$, and the Dirichlet conditions are assumed on the remaining part $\partial \Omega_{0}=\partial \Omega \backslash \partial \Omega_{1}$. In order to have a unified presentation, we introduce a function $\zeta$ assuming only the two values 0 and 1 such that $\left.\zeta\right|_{\partial \Omega_{1}}=1$ and $\left.\zeta\right|_{\partial \Omega_{0}}=0$. We then put

$$
\begin{aligned}
& \mathcal{B}_{u}(u)=\left(\frac{\partial u}{\partial v}+r_{1}(x) u\right) \zeta+(1-\zeta)\left(u-u_{0}\right) \\
& \mathcal{B}_{v}(v)=\left(\frac{\partial v}{\partial v}+r_{2}(x) v\right) \zeta+(1-\zeta)\left(v-v_{0}\right)
\end{aligned}
$$

Then the following conditions combine the aforementioned ones

$$
\mathcal{B}_{u}(u)=\mathcal{B}_{v}(v)=0, \quad \text { on } \partial \Omega \text {. }
$$

That weak solutions to weakly coupled elliptic systems are bounded has been well established using standard methods such as maximum principles, barrier functions, etc. However, these techniques are not available for (1.1). On the other hand, that bounded weak solutions to (1.1) are also classical solutions is still generally unknown. In fact, counterexamples in [8] confirmed that this is not the case for some strongly coupled systems, and that their bounded solution $(u, v)$ can only be partially regular. That is, there exists an open subset $\Omega_{0} \subset \Omega$ such that $(u, v)$ is Hölder continuous in $\Omega_{0}$. The Lebesgue measure of the singular set $\Omega \backslash \Omega_{0}$ is zero, and its Hausdorff dimension can be estimated (e.g., see [8]). It is now well known that, partial regularity is the best one can expect for general strongly coupled systems. Finding structural conditions for everywhere regularity is then an important problem. 
Our work is inspired by the following strongly coupled elliptic system

$$
(\mathrm{SKT}) \quad\left\{\begin{array}{l}
-\Delta\left[\left(\delta_{1}+a_{11} u+a_{12} v\right) u\right]=u\left(a_{1}-b_{1} u-c_{1} v\right), \\
-\Delta\left[\left(\delta_{2}+a_{21} u+a_{22} v\right) v\right]=v\left(a_{2}-b_{2} u-c_{2} v\right),
\end{array}\right.
$$

which was proposed by Shigesada, Kawasaki and Teramoto in [27] to study spatial segregation of two competing species. The Neumann boundary conditions were assumed.

Here, the constants $\delta_{i}, a_{i j}, a_{i}, b_{i}, c_{i}$ are positive. When $a_{i j}$ 's are all zero, (1.6) reduces to the well-studied Lotka-Voltera diffusion system. By introducing the self diffusion rates $a_{11}, a_{22}$ and the cross diffusion rates $a_{12}, a_{21}$, we take into account the diffusion pressures of each species creates on itself and the other. This system has drawn much attention recently (see e.g. [4, 12, 19-21, 24]) since an interesting pattern formation of coexistence may happen in contrast to the weakly coupled case.

System (1.6) is just a special case of (1.1) when $P^{u}, P^{v}, Q^{u}, Q^{v}$ are simply the partial derivatives of $P=\delta_{1} u+a_{12} u v+a_{11} u^{2}, Q=\delta_{2} v+a_{21} u v+a_{22} v^{2}$ with respect to $u, v$. One of the main vehicles in the aforementioned works is an a priori estimate of the $L^{\infty}$ norms of $u, v$. As one could reduce (1.6) to a weakly coupled system by making a change of variables $U=P$ and $V=Q$ (see e.g. $[19,24])$, maximum principles were used to achieve the desired $L^{\infty}$ estimates for $U, V$. Once the regularity of $U, V$ is established, the boundedness and regularity of $u, v$ follow immediately.

Unfortunately, this convenient change of variables is no longer available when system (1.1) is considered with $P^{u}, P^{v}, Q^{u}, Q^{v}$ being arbitrary functions in $u$ and $v$. Maximum principles or Harnack type inequalities, the key ingredients of the aforementioned works, are no longer available in this case. Recently, an elementary proof in [5] establishes $L^{\infty}$ bounds for positive solutions to systems similar to (1.1). Unfortunately, their proof, using straightforward integrations, only works when the domain $\Omega$ is a one-dimensional interval and the boundary condition is of Neumann type.

The first goal of this paper is to show that nonnegative weak solutions to (1.1) are bounded and Hölder continuous everywhere. This will be done in Section 2 , where we impose very general assumptions (see (A.1)-(A.3)) on the structure of the system and give a unified proof for all boundary condition types, including mixed boundary conditions (1.5). Our main idea is to construct a diffeomorphism $\vec{H}(u, v)$, being defined along the solution $(u, v)$, which links the structures of the two equations in a way that we can establish the boundedness and regularity of $\vec{H}(u, v)$. The boundedness and regularity of $\vec{H}$ then infer those of $u$ and $v$.

It is now known (see [8]) that Hölder continuous weak solutions of (1.1) also have Hölder continuous first derivatives. However, no estimates for their gradients have been discussed. In Section 2.2, we go further to estimate the Hölder norms of the gradients of the solutions. To achieve this, we will make use of the 
Morrey-Sobolev imbedding inequalities. Precisely, we shall prove in Section 2.2 that under the general assumptions (P.1), (P.2) (see Section 2) Hölder continuous weak solutions of (1.1) belong to $C^{\mu}(\Omega)$ for some $\mu>1$ (and are classical). Moreover, the norms $\|u\|_{C^{\mu}(\Omega)}$, $\|v\|_{C^{\mu}(\Omega)}$ can be controlled by a constant depending only on the parameters of the system. We also give a fairly general (easily verified in applications) condition (YYY) under which the discussed regularity properties and a priori estimates hold.

The estimate above is a crucial step that enables us to employ the fixed point theory to prove the existence of nonnegative solutions in the rest of this paper. In Section 3, we consider the existence of nonnegative solutions of (1.1), with nonhomogeneous mixed boundary conditions. The cornerstone of the proof is the existence of a compact operator mapping the positive cone of an ordered Banach space into itself, and its fixed points are classical solutions of (1.1). Under some general structure conditions for (1.1), see $\left(\mathrm{YYY}^{\prime}\right)$, the existence of such an operator is guaranteed. Using fixed point index theories, we establish the solvability of (1.1) in Corollary 3.2. To the best of our knowledge, this kind of result for nonhomogeneous mixed boundary conditions has never been addressed in the literature.

When the boundary conditions are homogeneous and the reaction terms are of some special forms, (1.1) possesses some "trivial" or "semi-trivial" solutions, which are not mathematically interesting. In applications, these solutions represent the washout (or wiped-out) states that are not desirable either. Whether there exists at least one non-trivial solution, or coexistence state, of (1.1) should be an interesting investigation.

Inspired by the pioneering works [19, 24], several papers have been devoted to the positive steady states of (1.6). Due to the non-variational nature of the system, degree theories (see $[4,5,26])$, bifurcation techniques (see $[10,11,28,29]$ ), and lower-upper solutions (see [23]) have been used to study (1.6). A common feature of these works (except [5]) is a rather tricky use of certain Harnack type inequality, which is available only for the special form (1.6) as in [19], to establish positive lower bounds for nontrivial solutions so that they are positive. The argument in [5, Lemma 1], using elementary ODE techniques, applies only for one dimensional domains. We should remark that none of these works discusses the mixed boundary conditions. The paper [26] also analyzes the Robin case but its argument makes use of the special structure of (1.6). Thus, all the aforementioned tools so far are neither available nor workable in our general settings.

To overcome such shortfalls, we will make use of the index apparatus developed in [15] (see also [7]) to compute the indices of semi-trivial solutions and shed light on the coexistence problem. Not only does our argument work for such setting, but it also unifies the treatments for different types of boundary conditions. Theorem 3.5 and Corollary 3.6 provide sufficient conditions in terms of certain principal eigenvalue problems for coexistence. Even though our method can handle much more general situations (see Remark 4.17), for comparison purposes, we 
will content ourself in Section 4 with the following case

$$
\begin{cases}P^{u}=\delta_{1}+a_{11} u+a_{12} v, & P^{v}=b_{11} u, \\ Q^{v}=\delta_{2}+a_{21} u+a_{22} v, & Q^{u}=b_{22} v,\end{cases}
$$

which generalizes (1.6) when $a_{12}=b_{11}$ and $a_{21}=b_{22}$.

Robin type conditions will be discussed in Section 4.1 while Neumann conditions will be the main topic of Section 4.2. For the Neumann case, we will discuss the effects of several types of self or cross diffusions on the coexistence. Noticeably, since we allow $a_{i j} \neq b_{i i}$, for $i \neq j$, we can introduce different types of cross diffusions. For example, one may refer to $a_{12}$, which goes with $v$, as the density cross diffusion; and $b_{11}$, which goes with $\nabla v$, as the gradient (or motility) cross diffusion. Our results then reveal interesting phenomena where these two kinds of cross diffusions can be very different. The analysis in Section 4.2 can apply to many other more general and interesting cases where different types of nonlinear dispersive forces due to the inter- and intra-specific interactions may give rise to a spatial segregation. Via the examples in Theorem 4.10, we provide a useful method to study such problems.

We conclude this work with some applications of our method to other problems. In Section 5, certain prey-predator and cooperative models in biology and ecology will be analyzed to demonstrate the generality of our results in Section 3 and Section 4.2.

Finally, we refer the reader who is interested in the parabolic counterpart of (1.1) to the works [16-18] for recent developments.

\section{A Priori Estimates}

2.1. Boundedness and Hölder regularity. In this section, we will extend the ideas in $[16,17]$ (for parabolic systems) to obtain the boundedness and Hölder continuity of the elliptic system (1.1). Following [16], for a given function $h \in$ $C^{1}\left(\mathbb{R}_{+}^{2}\right)$, we first consider the following first order partial differential equation

$$
H_{u}-h(u, v) H_{v}=0, \quad(u, v) \in \mathbb{R}_{+}^{2} .
$$

This equation for $H$ can be solved by the characteristic method (see [2, pp 97-99]) by considering the ODE system

$$
\vec{x}^{\prime}(t)=(1,-h), \quad \vec{p}^{\prime}(t)=\left(h_{u}, h_{v}\right) p_{v}, \quad z^{\prime}(t)=p_{u}-h p_{v}=0,
$$

where $\vec{x}(t)=(u(t), v(t)), \vec{p}(t)=\left(p_{u}(t), p_{v}(t)\right)=\left(H_{u}(\vec{x}(t)), H_{v}(\vec{x}(t))\right)$, $z(t)=H(\vec{x}(t))$.

The following result is elementary but useful. 
Lemma 2.1. Assume that $h \in C^{1}\left(\mathbb{R}_{+}^{2}\right)$ and that there exist positive constants $c_{1}$, $c_{2}, c_{3}>0$ such that

$$
\begin{gathered}
c_{1} \leq h(u, v) \leq c_{2}, \quad(u, v) \in \mathbb{R}_{+}^{2}, \\
\left|\int_{0}^{t} h_{v}(u(s), v(s)) \mathrm{d} s\right| \leq c_{3},
\end{gathered}
$$

along the solution $(u(t), v(t))$ of (2.2). Then there is a $C^{1}$ function $H$ satisfying (2.1) such that $H$ is defined on $\mathbb{R}_{+}^{2}$ and $H_{u}, H_{v}$ are positive, bounded from above and away from zero on $\mathbb{R}_{+}^{2}$.

Proof. We shall choose the initial data for $\vec{x}, \vec{p}$ on the line $Y=\{(u, v) \in$ $\left.\mathbb{R}_{+}^{2}: u=v\right\}$, which is non-characteristic, to be

$$
\begin{aligned}
& \vec{x}(0)=(u, u), \quad p_{u}(0)=h(u, u), \quad p_{v}(0)=1, \\
& \text { and } \quad H(u, v)=\int_{0}^{u} h(s, s) \mathrm{d} s+v .
\end{aligned}
$$

The argument in [16] then shows that $H(u(s), v(s))$ is well defined by being constant on the characteristic curves of (2.2). However, by (2.3), the characteristic curves defined by the first equation of (2.2) fill the first quadrant $\mathbb{R}_{+}^{2}$. Thus, $H$ is defined on $\mathbb{R}_{+}^{2}$. Concerning the last assertion, from the second equation of (2.2), we have

$$
p_{v}(t)=\exp \left(\int_{0}^{t} h_{v}(u(s), v(s)) \mathrm{d} s\right) .
$$

This and (2.4) give the boundedness of $H_{u}, H_{v}$.

We now go back to the system (1.1) and consider the following assumptions.

(A.1) $P^{u}, P^{v}, Q^{u}, Q^{v}$ are $C^{1}$ functions in $(u, v) \in \mathbb{R}_{+}^{2}$. There is a $\delta_{0}>0$ such that $P^{u} Q^{v}-P^{v} Q^{u}, P^{u}, Q^{v} \geq \delta_{0}$.

(A.2) There exists a constant $k>0$ such that the function $h$ defined by $h(u, v)=$ $\left(k P^{u}+Q^{u}\right) /\left(k P^{v}+Q^{v}\right)$ satisfies the conditions in Lemma 2.1.

(A.3) $f, g$ are continuous functions in $u, v$ and there exists a $K_{0}>0$ such that $k f(u, v)+g(u, v)<0$ whenever $u>K_{0}$ or $v>K_{0}$. Here, $k$ is the constant in (A.2).

Theorem 2.2. Suppose that (A.1)-(A.3) hold. Then nonnegative solutions for (1.1), with the mixed boundary condition (1.5), are bounded by a constant depending on $K_{0}$ and the diffusion parameters of the system.

Moreover, if (A.2) also holds for another positive constant $\bar{k} \neq k$, then $u, v$ are also Hölder continuous. Furthermore, for every $0<\mu<1,\|u\|_{C^{\mu}(\Omega)},\|v\|_{C^{\mu}(\Omega)}$ are bounded by a constant depending on $\mu$ and the parameters of the system. 
Proof. We assume the Robin boundary condition (1.2) and leave the general case to Remark 2.3. Multiplying the first equation by $k$ and adding the second equation, we get

$$
\nabla\left[\left(k P^{u}+Q^{u}\right) \nabla u+\left(k P^{v}+Q^{v}\right) \nabla v\right]=k f+g .
$$

Thanks to (A.2), we can use Lemma 2.1 to have a function $H=H(u, v)$ as a solution of (2.1). That is,

$$
\left(k P^{v}+Q^{v}\right) H_{u}=H_{v}\left(k P^{u}+Q^{u}\right) .
$$

Let $a_{k}(u, v)=\left(k P^{u}+Q^{u}\right) / H_{u}$. From (2.5) and (2.6), we have

$$
-\nabla\left(a_{k}(u, v) \nabla H\right)=F
$$

for $F=k f+g$. Testing this equation with $(H-K)_{+}$for any $K>0$, we obtain

$$
\begin{aligned}
\int_{\Omega} a_{k}(u, v)\left|\nabla(H-K)_{+}\right|^{2} \mathrm{~d} x-\int_{\partial \Omega} a_{k}(u, v) \frac{\partial H}{\partial v} & (H-K)_{+} \mathrm{d} \sigma \\
& =\int_{\Omega} F(H-K)_{+} \mathrm{d} x .
\end{aligned}
$$

From (A.1) and (A.2), it is easy to see that $k P^{u}+Q^{u}>0$. As $H_{u}>0$, we have that $a_{k}(u, v)>0$. On the other hand, from the boundary conditions for $u$, $v$, we have

$$
\frac{\partial H}{\partial v}=\frac{\partial H}{\partial u} \frac{\partial u}{\partial v}+\frac{\partial H}{\partial v} \frac{\partial v}{\partial v}=-r_{1}(x) \frac{\partial H}{\partial u} u(x)-r_{2}(x) \frac{\partial H}{\partial v} v(x) \leq 0 .
$$

Hence,

$$
0 \leq \int_{\Omega} F(H-K)_{+} \mathrm{d} x .
$$

Since $H_{u}, H_{v}$ are bounded from above and away from zero, we have $H(u, v)$ $\rightarrow+\infty$ iff $(u, v) \rightarrow \infty$. Therefore, there exists a $K_{1}>0$ such that $H(u, v)>K_{1}$ implies that $u$ or $v>K_{0}$. We choose $K=K_{1}$ in (2.8). Thanks to (A.3), we have $F(u, v)=k f(u, v)+g(u, v)<0$ when $H>K_{1}$. Hence, $F\left(H-K_{1}\right)_{+} \leq 0$ at $(u(x), v(x))$ for all $x \in \Omega$. This implies that the integral of the nonpositive function $F\left(H-K_{1}\right)_{+}$in $(2.8)$ is zero. Thus, $F\left(H-K_{1}\right)_{+} \equiv 0$. Again, since $F\left(H-K_{1}\right)_{+}<0$ if $H>K_{1}$, we must have that $H(u(x), v(x)) \leq K_{1}$. Clearly, $\left\{(u, v): H(u, v) \leq K_{1}\right\}$ is a bounded set. Hence, $u(x)$ and $v(x)$ are bounded by a constant $K_{2}$ depending on $K_{1}$ for all $x \in \Omega$. This constant $K_{2}$ obviously depends on $K_{0}$ and the parameters of the system defining the function $H$. 
We turn to the regularity assertion. Let us regard $W(x):=H(u(x), v(x))$ as a bounded weak solution to (2.7). Since $u(x), v(x)$ are now bounded, $k P^{u}+$ $Q^{u}, H_{u}$ are bounded from above and away from zero and so is the coefficient $\lambda(x)=a_{k}(u(x), v(x))$. Therefore, Equation (2.7) is uniformly elliptic. $H$ also satisfies the same type of boundary conditions for $u, v$. In fact, we can write $\partial H / \partial v+R(x) H=0$, where

$$
R(x):=\left(r_{1}(x) \frac{\partial H}{\partial u} u(x)+r_{2}(x) \frac{\partial H}{\partial v} v(x)\right) / H(u, v) .
$$

Here, by adding a constant to $H$, we can assume that $H$ is bounded away from zero.

The well-known regularity theory of elliptic equations $([9,13])$ gives us the Hölder regularity of $W$. That is, $W \in C^{\alpha}(\Omega)$ for some $\alpha \in(0,1)$. Moreover, as $a_{k}$ is continuous in $u, v$, we have that $\lambda(x)=a_{k}(u(x), v(x))$ is continuous in $x$. Again, the regularity theory of elliptic equations shows that $W \in C^{\alpha}(\Omega)$ for all $\alpha \in(0,1)$.

If (A.2) holds for some $\bar{k}>0$, the argument above shows that the corresponding function $\bar{H}$ is also Hölder continuous. Let $\vec{H}=(H, \bar{H})$. Using (2.6), we compute its Jacobian

$$
\left|J_{\vec{H}}\right|=H_{u} \bar{H}_{v}-H_{v} \bar{H}_{u}=\frac{H_{v} \bar{H}_{v}(k-\bar{k})\left(P^{u} Q^{v}-P^{v} Q^{u}\right)}{\left(k P^{v}+Q^{v}\right)\left(\bar{k} P^{v}+Q^{v}\right)} .
$$

Thanks to (A.1), (A.2) and Lemma 2.1, $\left|J_{\vec{H}}\right|$ is bounded from above and away from zero. Therefore, $\vec{H}: \mathbb{R}_{+}^{2} \rightarrow \mathbb{R}_{+}^{2}$ is a diffeomorphism. Since $H$ and $\bar{H}$ are Hölder continuous, so is $(u, v)$.

Remark 2.3. The proof above also works for the mixed boundary condition case. In fact, if we also require that $K_{1}$ be larger than $\max _{\partial \Omega_{0}} H\left(u_{0}, v_{0}\right)$, where $u_{0}$, $v_{0}$ are the (bounded) boundary values of $u$, $v$, then $\left.\left(H-K_{1}\right)_{+}\right|_{\partial \Omega_{0}} \equiv 0$ and the boundary integral resulting in the integration by parts of the proof is still negative. The proof then goes on as before, if we also assume that $u_{0}, v_{0}$ are $C^{1}$ functions on $\partial \Omega$. Note also that $H$ satisfies a similar mixed boundary condition.

We conclude this section by a simple application of Theorem 2.2. Apparently, the conditions (A.1), (A.2) seem to be the most important ones to be verified. We consider the following situation, which is inspired by and generalizes the Shigesada, Kawasaki and Teramoto system (1.6).

(YYY) For some integer $N \geq 1$, assume that $P^{u}, P^{v}, Q^{u}, Q^{v}$ are polynomials of order $N$ of the form

$$
\sum_{i+j \leq N} a_{i j} u^{i} v^{j}
$$


where $a_{i j}$ are nonnegative constants. Furthermore, we assume that the coefficients $a_{00}, a_{0 N}, a_{N 0}$ of $P^{u}$ and $Q^{v}$ are positive, and $P^{u} Q^{v}-P^{v} Q^{u}>$ $\delta_{0}>0$ on $\mathbb{R}_{+}^{2}$.

Obviously, this condition guarantees (A.1). Let us verify (A.2). To this end, let $k>0$ be given. We consider the function

$$
h(u, v)=\frac{k P^{u}+Q^{u}}{k P^{v}+Q^{v}}=\frac{R(u, v)}{S(u, v)},
$$

where $R, S$ are polynomials of order $N$ in $(u, v)$ with nonnegative coefficients. Moreover, the free constants and the coefficients of $u^{N}, v^{N}$ in these polynomials are positive. A simple use of Young's inequality shows that $h(u, v)$ is bounded from above and away from zero. Thus, (2.3) of Lemma 2.1 is verified.

We now turn our attention to (2.4). First of all, we have

$$
\left|h_{v}(u, v)\right|=\left|\frac{R_{v} S-R S_{v}}{S^{2}}\right| \leq \frac{T(u, v)}{S^{2}(u, v)},
$$

where $T(u, v)$ is a polynomial of order $2 N-1$. Hence, $(1+u+v) T(u, v)$ is a polynomial of order $2 N$. Since $S^{2}$ is of order $2 N$ and its coefficients for $u^{2 N}$, $v^{2 N}$, as well as the free constant, are positive, it is easy to see that there is a positive constant $C_{2}$ such that

$$
\frac{(1+u+v) T(u, v)}{S^{2}(u, v)} \leq C_{2} \Rightarrow\left|h_{v}(u, v)\right| \leq \frac{C_{2}}{1+u+v} .
$$

Let $\left(u_{*}, v_{*}\right)$ be a point on $\Upsilon$. We consider the characteristic curve $(u(t), v(t))$ emanating from this point. From (2.3) and the first equation in (2.2), there must be some constant $C_{0}>0$ so that if $(u(t), v(t))$ stays in $\mathbb{R}_{+}^{2}$, then $|t| \leq C_{0} u_{*}$ and either $u(t) \geq u_{*}$ or $v(t) \geq v_{*}=u_{*}$, along this characteristic curve. Putting these together, we have

$$
\begin{aligned}
\left|\int_{0}^{t} h_{v}(u(s), v(s)) \mathrm{d} s\right| & \leq \int_{-|t|}^{|t|} \frac{C_{1}}{1+u(s)+v(s)} \mathrm{d} s \\
& \leq \int_{0}^{C_{0} u_{*}} \frac{C_{2}}{1+u_{*}} \mathrm{~d} s \leq C_{4}
\end{aligned}
$$

for some constant $C_{4}$ depending only on $k$ and the coefficients $a_{i j}$ in (2.9). This shows that (YYY) verifies (A.1) and (A.2).

The argument above gives the following result.

Theorem 2.4. Assume that (1.1), with the mixed boundary condition (1.5), satisfies the structural conditions (A.3) and (YYY). Let $(u, v)$ be a nonnegative solution to (1.1). Then, for every $0<\mu<1,\|u\|_{C^{\mu}(\Omega)},\|v\|_{C^{\mu}(\Omega)}$ are bounded by a constant depending on $\mu$ and the parameters of the system. 
Remark 2.5. From the argument above, it is clear that the assumption $a_{i j} \geq$ 0 in (YYY) can be relaxed. Theorem 2.4 remains valid if one can find two positive reals $k, \bar{k}$ such that the polynomials $R(u, v), S(u, v)$ (defined in (2.10)) are positive on $\mathbb{R}_{+}^{2}$, and their coefficients of $u^{N}, v^{N}$ and the free constants are also positive.

Remark 2.6. Several works concern a simplified version of the (SKT) system (1.6) where $a_{11}=a_{22}=0$. This system is a special case of (1.1) where $P^{u}, Q^{u}$ (respectively, $P^{v}, Q^{v}$ ) are functions in $v$ (respectively, $u$ ) only. In this case, the assumption (2.3) on $h$ of Lemma 2.1 does not hold as

$$
h(u, v)=\frac{k P^{u}(v)+Q^{u}(v)}{k P^{v}(u)+Q^{v}(u)}
$$

is no longer bounded on $\mathbb{R}_{+}^{2}$. However, the the function $H$ in the proof of Theorem 2.4 is now easy to find and the proof can be repeated with minor modifications. In fact, in order to verify (2.6), we need only take

$$
H(u, v)=\int_{0}^{u} \frac{d s}{k P^{v}(s)+Q^{v}(s)}+\int_{0}^{v} \frac{d s}{k P^{u}(s)+Q^{u}(s)} .
$$

The only assumptions we need for the boundedness of $u, v$ are:

(1) $a_{k}(u, v)=\left(k P^{u}(v)+Q^{u}(v)\right) / H_{u} \geq 0$;

(2) $H(u, v) \rightarrow \infty$ iff $(u, v) \rightarrow \infty$.

The first condition holds if $\left(k P^{u}(v)+Q^{u}(v)\right) /\left(k P^{v}(u)+Q^{v}(u)\right) \geq 0$ for $u$, $v \geq 0$. The second condition holds, at least, for the case when $P^{u}, P^{v}, Q^{u}, Q^{v}$ are linear functions with positive coefficients and satisfy (A.1). Indeed, we then have $H(u, v)=\alpha \log (a+b u)+\beta \log (c+d u)$, with $\alpha, \beta, a, b, c, d$ being positive. Thus, $u, v$ are bounded in this case. Once this boundedness is established, the proof of Hölder continuity goes on as before.

2.2. Uniform Hölder bounds for the gradients. In this section we estimate the Hölder norm of the gradients of the solutions for (1.1). These estimates provide the compactness of the fixed point maps in the study of coexistence solutions. In fact, we will be able to consider much more general systems of the form

$$
-\operatorname{div}(a(\vec{u}) \nabla \vec{u})=\vec{F}(\vec{u}),
$$

where $\vec{u}=\left(u_{1}, u_{2}, \ldots, u_{m}\right)$ is a vector valued function and $a(\vec{u})$ is a $m \times m$ matrix. Let $r_{i}(x)$ 's be $C^{1}$ functions on $\bar{\Omega}$. We also assume the Robin boundary condition

$$
\frac{\partial u_{i}}{\partial v}+r_{i}(x) u_{i}=0
$$

and leave the mixed boundary condition (1.5) case to Remark 2.13.

We consider the following structural conditions: 
(P.1) There exists a positive constant $\lambda$ such that

$$
\lambda|\xi|^{2} \leq a(\vec{u}) \xi_{i} \xi_{j}, \quad \text { for any } \xi \in \mathbb{R}^{m} .
$$

(P.2) There exists a continuous function $C_{0}(\vec{u})$ such that: $|a(\vec{u})|,\left|a_{\vec{u}}(\vec{u})\right|$, $|\vec{F}(\vec{u})| \leq C_{0}(|\vec{u}|)$.

Theorem 2.7. Assume (P.1) and (P.2). Let $\vec{u}$ be a solution for (2.11). Suppose that $\vec{u}$ is Hölder continuous and there exists a constant $C_{\alpha}$ such that:

$$
\|\vec{u}\|_{C^{\alpha}} \leq C_{\alpha}, \quad \text { for all } \alpha \in(0,1) .
$$

Then there exist $\alpha<1$ and a positive constant $C\left(\mu, \lambda, C_{\alpha}, C_{0}\left(\|u\|_{\infty}\right)\right)$, for any $\mu \in(1,2)$, such that $\vec{u} \in C^{\mu}(\Omega)$ and

$$
\|\vec{u}\|_{C^{\mu}(\Omega)} \leq C\left(\mu, \lambda, C_{\alpha}, C_{0}\left(\|u\|_{\infty}\right)\right) .
$$

This general result and Theorem 2.4 immediately give us a stronger result.

Corollary 2.8. Assume that (1.1), with the mixed boundary condition (1.5), satisfies the structural conditions (A.3) and (YYY). Let $(u, v)$ be a nonnegative solution to (1.1). For any $\mu \in(1,2),\|u\|_{C^{\mu}(\Omega)},\|v\|_{C^{\mu}(\Omega)}$ are bounded by a constant depending on $\mu$ and the parameters of the system.

In the proof below, we will only deal with the Neumann boundary condition $(r(x)=0)$, and leave the Robin case to Remark 2.12.

The main idea of the proof of this theorem is to use the imbedding results for Morrey's spaces. We recall the definitions of the Morrey space $M^{p, \lambda}(\Omega)$ and the Sobolev-Morrey space $W^{1,(p, \lambda)}$. Let $B_{R}(x)$ be a ball centered at $x$ with radius $R$ in $\mathbb{R}^{n}$.

We say that $f \in M^{p, \lambda}(\Omega)$ if $f \in L^{p}(\Omega)$ and

$$
\|f\|_{M^{p, \lambda}}^{p}:=\sup _{x \in \Omega, \rho>0} \rho^{-\lambda} \int_{B_{\rho}(x) \cap \Omega}|f|^{p} \mathrm{~d} y<\infty .
$$

Moreover, $f$ is in Sobolev-Morrey space $W^{1,(p, \lambda)}$ if $f \in W^{1, p}(\Omega)$ and

$$
\|f\|_{W^{1,(p, \lambda)}}^{p}:=\|f\|_{M^{p, \lambda}}^{p}+\|\nabla f\|_{M^{p, \lambda}}^{p}<\infty .
$$

If $\lambda<n-p, p \geq 1$, and $p_{\lambda}=p(n-\lambda) /(n-\lambda-p)$, we then have the following imbedding result (see Theorem 2.5 in [6])

$$
W^{1,(p, \lambda)}(B) \subset M^{p_{\lambda}, \lambda}(B)
$$

and if $\lambda>n-p$ and $\alpha_{\lambda}=(\lambda-(n-p)) / p$,

$$
W^{1,(p, \lambda)}(B) \subset C^{0, \alpha_{\lambda}}(\bar{B}) .
$$


We then proceed by proving some estimates for the Morrey norms of the gradients of the solutions. From now on, let us fix a point $x \in \Omega$. As far as no ambiguity can arise, we write $B_{R}=B_{R}(x)$ and $\Omega_{R}=\Omega \cap B_{R}$.

We first have the following technical lemma.

Lemma 2.9. Assume the conditions of Theorem 2.7. For sufficiently small $R>0$, we have the following estimate

$$
\int_{\Omega_{R}}|\nabla \vec{u}|^{2} \mathrm{~d} x+\int_{\Omega_{R}}|\Delta \vec{u}|^{2} \mathrm{~d} x \leq C\left(C_{\alpha}\right) R^{n-2+2 \varepsilon},
$$

for $\varepsilon=2 \alpha-1$. Here $\Delta \vec{u}=\left(\Delta u_{1}, \Delta u_{2}, \ldots, \Delta u_{m}\right)$.

In the proof below, we will need the following useful result by Ladyzhenskaja et al. [14]. The result was stated for the scalar function $u$ but the same proof applies for vector-valued functions. Note also that the condition $u \eta=0$ on $\partial \Omega$ in [14, Lemma II.5.4] can be replaced by $(\partial u / \partial v) \eta=0$ in order that the calculation in the proof of that lemma, using integration by parts, may continue.

Lemma 2.10 ([14, Lemma II.5.4]). For any function $\vec{u}$ in $W^{1,2 s+2}\left(\Omega, \mathbb{R}^{m}\right)$ and smooth real-valued function $\eta$ such that either $\vec{u} \eta$ or $(\partial \vec{u} / \partial v) \eta$ vanishes on $\partial \Omega$, we have

$$
\begin{aligned}
& \int_{\Omega}|\nabla \vec{u}|^{2 s+2} \eta^{2} \mathrm{~d} x \\
& \quad \leq \operatorname{osc}^{2}\{\vec{u}, \Omega\} \operatorname{Cont} \int_{\Omega}\left(|\nabla \vec{u}|^{2 s-2}|\Delta \vec{u}|^{2} \eta^{2}+|\nabla \vec{u}|^{2 s}|\nabla \eta|^{2}\right) \mathrm{d} x .
\end{aligned}
$$

Proof of Lemma 2.9. Let $\eta(x)$ be a cut-off function for $B_{R}$ and $B_{2 R}$, that is, $\eta=1$ on $B_{R}$ and $\eta=0$ outside of $B_{2 R}$.

We first test the $i^{\text {th }}$ equation of (2.11) with $\left(\vec{u}-\vec{u}_{R}\right){ }_{i} \eta^{2}$. Integrating by parts and summing the results, we easily derive (thanks to (2.12))

$$
\lambda \int_{\Omega_{2 R}}|\nabla \vec{u}|^{2} \eta^{2} \mathrm{~d} x \leq \int_{\Omega_{2 R}}\left[\left(\vec{u}-\vec{u}_{R}\right)^{T} a(\vec{u}) \nabla u \eta \nabla \eta+\left(\vec{u}-\vec{u}_{R}\right)^{T} \vec{F}(\vec{u}) \eta^{2}\right] \mathrm{d} x .
$$

Since $\vec{u}$ is Hölder continuous, $\left|\vec{u}-\vec{u}_{R}\right| \leq C_{\alpha} R^{\alpha}$. Using Young's inequality and the facts that $|a(\vec{u})|,|\vec{F}(\vec{u})| \leq C,|D \eta| \leq 1 / R$, we obtain (for small $R$ )

$$
\int_{\Omega_{R}}|\nabla \vec{u}|^{2} \mathrm{~d} x \leq \frac{C}{R^{2}} \int_{\Omega_{2 R}}\left|\vec{u}-\vec{u}_{R}\right|^{2} \mathrm{~d} x+C R^{n} \leq C R^{n-2+2 \alpha} .
$$

Rewrite (2.11) as

$$
-a(\vec{u}) \Delta \vec{u}=a_{\vec{u}}(\vec{u}) \nabla \vec{u} \bullet \nabla \vec{u}+\vec{F}(\vec{u}),
$$


and test this with $-\Delta \vec{u} \eta^{2}$ to get

$$
\int_{B_{2 R}}|\Delta \vec{u}|^{2} \eta^{2} \mathrm{~d} x \leq C \int_{B_{2 R}}|\nabla \vec{u}|^{4} \eta^{2} \mathrm{~d} x+C R^{n} .
$$

Here, we have just used (P.1), (P.2) and Young's inequality. We apply Lemma 2.10, with $s=1$, to the integral on the right hand side of (2.17) to get

$\int_{\Omega_{2 R}}|\Delta \vec{u}|^{2} \eta^{2} \mathrm{~d} x \leq C R^{2 \alpha} \int_{\Omega_{2 R}}|\Delta \vec{u}|^{2} \eta^{2} \mathrm{~d} x+C R^{2 \alpha} \int_{\Omega_{2 R}}|\nabla \vec{u}|^{2}|\nabla \eta|^{2} \mathrm{~d} x+C R^{n}$.

Choosing $R$ sufficiently small in this inequality and using the estimate (2.16), we obtain

$$
\int_{B_{R}}|\Delta \vec{u}|^{2} \mathrm{~d} x \leq C R^{n-4+4 \alpha} .
$$

Let $\varepsilon=2 \alpha-1$. The estimate above and (2.16) give the lemma.

We are now ready to give the following proof:

Proof of Theorem 2.7. We proved, for any $R$ small and $\varepsilon=2 \alpha-1$, that

$$
\int_{\Omega_{R}}|\nabla \vec{u}|^{2} \mathrm{~d} x, \quad \int_{\Omega_{R}}|\Delta \vec{u}|^{2} \mathrm{~d} x \leq C R^{n-2+2 \varepsilon} .
$$

We now apply [22, Lemma 4.1] to assert that the norms of $\nabla u$ in $W^{1,(2, \lambda)}\left(\Omega_{R}\right)$, $\lambda=n-2+2 \varepsilon$ are bounded. Therefore, by the imbedding inequality (2.14), we obtain the boundedness of $\|\nabla \vec{u}\|_{C^{\mu}(\Omega)}, \mu=\varepsilon$. Since $\alpha$ can be close to 1 , we can make $\mu$ close to 1 . The proof is then completed.

Remark 2.11. It is easy to see that the same proof applies to the system

$$
-\operatorname{div}(a(\vec{u}) \nabla \vec{u})=\vec{F}(\vec{u}, \nabla \vec{u}),
$$

where $\vec{F}(\vec{u}, \nabla \vec{u})$ satisfies $|\vec{F}(\vec{u}, \nabla \vec{u})| \leq C_{0}(|\vec{u}|)\left(1+|\nabla \vec{u}|^{2}\right)$ for some continuous function $C_{0}$.

Remark 2.12. The case of Robin boundary conditions can be reduced to the Neumann case by a simple change of variables. First of all, since our proof is based on the local estimate of Lemma 2.9, we need only to study the inequalities of its proof near the boundary. As $\partial \Omega$ is smooth, we can locally flatten the boundary and assume that $\partial \Omega$ is the plane $\left\{x_{n}=0\right\}$. Furthermore, we can take $\Omega_{R}=$ $\left\{\left(x^{\prime}, x_{n}\right): x_{n}>0,\left|\left(x^{\prime}, x_{n}\right)\right|<R\right\}$. The boundary conditions become

$$
\frac{\partial u_{i}}{\partial x_{n}}+\tilde{r}_{i}\left(x^{\prime}\right) u_{i}=0
$$


We then introduce $U\left(x^{\prime}, x_{n}\right)=\left(U_{1}\left(x^{\prime}, x_{n}\right), \ldots, U_{m}\left(x^{\prime}, x_{n}\right)\right)$ with

$$
U_{i}\left(x^{\prime}, x_{n}\right)=\exp \left(x_{n} \tilde{r}_{i}\left(x^{\prime}\right)\right) u_{i}\left(x^{\prime}, x_{n}\right) .
$$

Obviously, $U$ satisfies the Neumann boundary condition on $x_{n}=0$. Simple calculations also show that $U$ verifies a system similar to that for $u$, and the conditions (P.1) and (P.2) are still valid. In fact, there will be some extra terms occurring in the divergence parts of the equations for $U$, but these terms can be handled by a simple use of Young's inequality so that our proof is still in force. Thus Theorem 2.7 applies to $U$, and the estimates for $u$ then follow.

Remark 2.13. Concerning the nonhomogeneous mixed boundary conditions (1.5), Theorem 2.7 holds if we assume that $\left.\vec{u}\right|_{\partial \Omega_{0}}$ is the trace on $\partial \Omega$ of some $\varphi \in C^{2}\left(\Omega, \mathbb{R}^{m}\right)$ and $\varphi$ satisfies the same boundary conditions. To see this, we first reduce the nonhomogeneous boundary conditions to the homogeneous ones by considering $U=\vec{u}-\varphi$. From (2.11), the system for $U$ is

$$
-\operatorname{div}(a(\vec{u}) \nabla U)=a(\vec{u})_{\vec{u}} \nabla U \nabla \varphi+a_{\vec{u}}(\vec{u}) \nabla \varphi \nabla \varphi+a(\vec{u}) \Delta \varphi+F(\vec{u}) .
$$

Noticing that the right-hand side satisfies the condition in Remark 2.11, we can repeat the proof above. In fact, we need only rework the proof of Lemma 2.9 for the case $B_{R}$ intersects the boundary $\partial \Omega$. From Remark 2.12, we can also assume the $r_{i} \equiv 0$. We keep in mind that Lemma 2.10 is still available as $\left.U\right|_{\partial \Omega}=$ 0 or $(\partial U / \partial v) \eta=0$. In this case, we test the above system with $U \eta^{2}$. Since $|U(x)| \leq C_{\alpha} R^{\alpha}$ for $x \in B_{R}$, simple uses of Young's inequality will show that similar estimates to (2.16), (2.17) for $\nabla \vec{u}, \Delta \vec{u}$ hold for $\nabla U, \Delta U$ as well. Thus, the proof above can go on as before.

We conclude this section by presenting a simple application of Corollary 2.8 to the generalized version of the (SKT) system (1.6) (see also Remark 2.6). We consider

$$
\left\{\begin{array} { l } 
{ P ^ { u } = \delta _ { 1 } + a _ { 1 1 } u + a _ { 1 2 } v , } \\
{ P ^ { v } = b _ { 1 1 } u , } \\
{ Q ^ { v } = \delta _ { 2 } + a _ { 2 1 } u + a _ { 2 2 } v , } \\
{ Q ^ { u } = b _ { 2 2 } v , }
\end{array} \text { and } \left\{\begin{array}{l}
f(u, v)=u\left(a_{1}-b_{1} u-c_{1} v\right), \\
g(u, v)=v\left(a_{2}-b_{2} u-c_{2} v\right) .
\end{array}\right.\right.
$$

Estimates for the $L^{\infty}$ norm of $u, v$ for the case $a_{12}=b_{11}$ and $a_{21}=b_{22}$ (and Neumann boundary conditions) were done in [19, Lemma 2.3] via maximum principle arguments and a simple change of variables. In [19], due to the special structure of (1.6), such $L^{\infty}$ estimates are sufficient for their analysis. Of course, the techniques in $[10,19,24,25,29]$ cannot apply here because such a change of variables is no longer possible. In the present work, we need only to assume that

(B.1) $\delta_{i}, a_{i}, b_{i}, c_{i}>0$ and $a_{i j} \geq 0$ such that $a_{11}, a_{12}+b_{22}, a_{21}+b_{11}, a_{22}>0$. Furthermore, $\left(\sqrt{a_{11} a_{22}}+\sqrt{a_{12} a_{21}}\right)^{2} \geq b_{11} b_{22}$. 
For any $(u, v) \in \mathbb{R}_{+}^{2}$, simple calculations show that

$$
\frac{\min \left\{\delta_{1}, a_{11}, a_{12}+b_{22}\right\}}{\max \left\{\delta_{2}, a_{22}, a_{21}+b_{11}\right\}} \leq h(u, v):=\frac{P^{u}+Q^{u}}{P^{v}+Q^{v}} \leq \frac{\max \left\{\delta_{1}, a_{11}, a_{12}+b_{22}\right\}}{\min \left\{\delta_{2}, a_{22}, a_{21}+b_{11}\right\}},
$$

and

$$
\begin{aligned}
& P^{u} Q^{v}-P^{v} Q^{u} \\
& \quad \geq \delta_{1} \delta_{2}+a_{11} a_{21} u^{2}+\left(a_{11} a_{22}+a_{12} a_{21}\right) u v+a_{12} a_{22} v^{2}-b_{11} b_{22} u v \\
& \quad \geq \delta_{1} \delta_{2}+\left[\left(\sqrt{a_{11} a_{22}}+\sqrt{a_{12} a_{21}}\right)^{2}-b_{11} b_{22}\right] u v \geq \delta_{1} \delta_{2} .
\end{aligned}
$$

Thus, (B.1) verifies Remark 2.5 with $k=1$. It is easy to see that the same claim holds for some $\bar{k}$ sufficiently close to 1 . The condition (A.3) is trivially satisfied by such choice of $f, g$. We see that Corollary 2.8 applies in this case. We would like to remark that negative $b_{i i}$ 's are allowed here.

Corollary 2.14. Assume that (1.1), with the mixed boundary condition (1.5), satisfies the structural conditions (2.18) and (B.1). Let $(u, v)$ be a nonnegative solution to (1.1). There exists $\mu>1$ such that $\|u\|_{C^{\mu}(\Omega)},\|v\|_{C^{\mu}(\Omega)}$ are bounded by a constant depending on the parameters of the system.

Obviously, Corollary 2.8 is general enough to cover several important applications, and its a-priori estimates for higher norms will play a crucial role in other investigations of (1.1). In the next section, our analysis of the coexistence problem for the generalized (SKT) systems above will rely heavily on this result.

\section{The Fixed Point Map and Its Indices}

In this section, we study the solvability for the general system (1.1) with the nonhomogeneous mixed boundary condition (1.5). Since this system is not variational, index theory will be employed here. We refer the reader to [1, Section 11] for the definition and basic properties of the fixed point index theory. On the other hand, as we will study in Section 4 the existence of positive solutions when (1.1) satisfies $\left(Y_{Y} Y^{\prime}\right)$ below, we also prepare some index results for that problem.

In certain cases, trivial (or washout) solutions may already exist. Thus, we will seek other (or coexistence) solutions. The main tools of our investigation are the fixed point index-theoretic apparatus devised in [15]. To proceed, we first construct a compact map $T$ in an ordered Banach space $X$ such that its fixed points are solutions for (1.1). Then, we compute the fixed-point index of $T$ in a subset $U$ of the positive cone $X_{+}$of $X$, and the sum of fixed-point indices of the "trivial" and "semi-trivial" fixed points of $T$ in $U$. If the two computed numbers are different, then there exists at least one more fixed point of $T$ inside $U$, which is a positive solution for (1.1).

For triangular systems (see [15]) or the original (SKT) (1.6) (see $[19,24]$ ), it is quite easy and natural to define the map $T$, on the space $C^{0}$ of continuous 
functions, such that the scheme above is applicable. This, however, is not the case for the general system (1.1) considered in this paper. Throughout this section, we will assume the following structure, which is a little more restrictive than (YYY) regarding the forms of the cross diffusions $P^{v}, Q^{u}$.

$\left(Y Y Y^{\prime}\right)$ The functions $P^{u}, P^{v}, Q^{u}, Q^{v}$ satisfy (YYY) and (A.3). Furthermore, we assume that

$$
P^{v}(u, v)=u \tilde{P}^{v}(u, v), \quad Q^{u}(u, v)=v \tilde{Q}^{u}(u, v)
$$

where $\tilde{P}^{v}, \tilde{Q}^{u}$ are polynomials. Moreover, we suppose that $f(u, v)=$ $u \tilde{f}(u, v)$ and $g(u, v)=u \tilde{g}(u, v)$ for some continuous functions $\tilde{f}, \tilde{g}$ such that $f, g$ satisfy (A.3).

Under this assumption, via Corollary 2.8 , the $C^{\mu}$ norms $(\mu \in(1,2))$ of the nonnegative solutions of (1.1) are uniformly bounded. Our main task is to determine a suitable fixed-point map $T$ and the working functional space $X$.

3.1. Construction of the compact operator. The system (1.1) can be rewritten as:

$$
\left\{\begin{array}{r}
-\left(P^{u} \Delta u+P^{v} \Delta v\right)-\left(P_{u}^{u}|\nabla u|^{2}+P_{v}^{v}|\nabla v|^{2}\right) \\
-\left(P_{v}^{u}+P_{u}^{v}\right) \nabla u \nabla v+\gamma u=f(u, v)+\gamma u, \\
-\left(Q^{u} \Delta u+Q^{v} \Delta v\right)-\left(Q_{v}^{v}|\nabla v|^{2}+Q_{u}^{u}|\nabla u|^{2}\right) \\
-\left(Q_{v}^{u}+Q_{u}^{v}\right) \nabla u \nabla v+\gamma v=g(u, v)+\gamma v,
\end{array}\right.
$$

where we just added $\gamma u, \gamma v$, with $\gamma \in[0,1]$, to both sides of the equations. The introduction of this parameter $\gamma$ will be clear later, when we discuss the fixed-point index results.

Let us denote

$$
\Theta:=\left(\begin{array}{ll}
P^{u} & P^{v} \\
Q^{u} & Q^{v}
\end{array}\right), \quad d(\vec{u})=\operatorname{det}(\Theta)=P^{u} Q^{v}-P^{v} Q^{u} .
$$

Let $k$ be a positive constant, which is to be determined later. We multiply $d(\vec{u}) \Theta^{-1}$ to $(3.2)$ and then add $k u, k v$ to the equations. We obtain

$$
\left\{\begin{array}{l}
-d(\vec{u}) \Delta u-d_{11}(\vec{u}) \nabla u+\left(d_{12}(\vec{u})+c_{1, \gamma}+k\right) u=F(\vec{u}), \\
-d(\vec{u}) \Delta v-d_{21}(\vec{u}) \nabla v+\left(d_{22}(\vec{u})+c_{2, \gamma}+k\right) v=G(\vec{u}),
\end{array}\right.
$$


where (notice (3.1))

$$
\left\{\begin{array}{l}
d_{11}(\vec{u})=\left(P_{u}^{u} Q^{v}-P^{v} Q_{u}^{u}\right) \nabla u+\left(P_{v}^{u}+P_{u}^{v}\right) Q^{v} \nabla v-\left(Q_{v}^{u}+Q_{u}^{v}\right) P^{v} \nabla v \\
d_{21}(\vec{u})=\left(Q_{v}^{v} P^{u}-Q^{u} P_{v}^{v}\right) \nabla v+\left(Q_{v}^{u}+Q_{u}^{v}\right) P^{u} \nabla u-\left(P_{v}^{u}+P_{u}^{v}\right) Q^{u} \nabla u, \\
d_{12}(\vec{u})=\left(Q_{v}^{v} \tilde{P}^{v}-Q^{v} \tilde{P}_{v}^{v}\right)|\nabla v|^{2}, \\
c_{1, \gamma}(\vec{u})=\left(Q^{v}-\tilde{P}^{v} v\right) \gamma \\
d_{22}(\vec{u})=\left(P_{u}^{u} \tilde{Q}^{u}-P^{u} \tilde{Q}_{u}^{u}\right)|\nabla u|^{2}, \\
c_{2, \gamma}(\vec{u})=\left(P^{u}-\tilde{Q}^{u} u\right) \gamma
\end{array}\right.
$$

and

$$
\left(\begin{array}{l}
F(\vec{u}) \\
G(\vec{u})
\end{array}\right)=\left(\begin{array}{l}
\tilde{F}(\vec{u})+k u \\
\tilde{G}(\vec{u})+k v
\end{array}\right)
$$

with

$$
\left(\begin{array}{l}
\tilde{F}(\vec{u}) \\
\tilde{G}(\vec{u})
\end{array}\right)=d(\vec{u}) \Theta^{-1}\left(\begin{array}{l}
f(u, v)+\gamma u \\
g(u, v)+\gamma v
\end{array}\right) .
$$

For $i \in\{1,2\}$, we define $X^{i}=C^{1}(\Omega) . X^{i}$ is then a Banach space with the norm $\|u\|_{X^{i}}:=\|u\|_{C^{1}(\Omega)}$. We see that $X^{1}, X^{2}$ are ordered Banach spaces with the positive cones $X_{+}^{i}=\left\{u \in X^{i}: u(x) \geq 0, \forall x \in \Omega\right\}$. Let $X=X^{1} \times X^{2}$ and $\|(u, v)\|_{X}=\|u\|_{X^{1}}+\|v\|_{X^{2}}$. Then $X$ is an ordered Banach space with the positive cone $X_{+}=X_{+}^{1} \times X_{+}^{2}$.

Let $\mu>1$ be as in Corollary 2.14. From this result, there is $R_{0}>0$ depending only on the parameters of (1.1) such that the $C^{\mu}$ norms of its nonnegative solutions are bounded by $R_{0}$. Let $R_{1}=2 R_{0}+1$. We consider the set

$$
U:=\left\{(\psi, \varphi) \in X_{+}:\|(\psi, \varphi)\|_{X}<R_{1}\right\} .
$$

For each $(\psi, \varphi) \in U$ and for sufficiently large $k$, we define

$$
\begin{aligned}
& K_{i, \gamma}(\psi, \varphi) \\
&=\left\{-d(\psi, \varphi) \Delta \bullet-d_{i 1}(\psi, \varphi) \nabla \bullet+\left(d_{i 2}(\psi, \varphi)+c_{i, \gamma}(\psi, \varphi)+k\right) \bullet,\right. \\
&\left.\mathcal{B}_{i}(\bullet)\right\}^{-1},
\end{aligned}
$$

where $\mathcal{B}_{1}=\mathcal{B}_{u}$ and $\mathcal{B}_{2}=\mathcal{B}_{v}$. Then $K_{i, \gamma}$ is a compact map from $C^{1}(\Omega)$ (or even $\left.C^{0}(\Omega)\right)$ into itself. In fact, if $h$ is a function in $C^{1}(\Omega)$, then $u=K_{i, y}(h)$ is just the solution of

$$
\left\{\begin{array}{l}
-d(\psi, \varphi) \Delta u-d_{i 1}(\psi, \varphi) \nabla u+\left(d_{i 2}(\psi, \varphi)+c_{i, \gamma}(\psi, \varphi)+k\right) u=h, \\
\mathcal{B}_{i}(u)=0
\end{array}\right.
$$


We also put $K_{i}=K_{i, 0}$, that is

$$
K_{i}(\psi, \varphi)=\left\{-d(\psi, \varphi) \Delta \bullet-d_{i 1}(\psi, \varphi) \nabla \bullet+\left(d_{i 2}(\psi, \varphi)+k\right) \bullet, \mathcal{B}_{i}(\bullet)\right\}^{-1}
$$

The operators above are well defined if $k$ is sufficiently large. In fact, since $(\psi, \varphi) \in U$, the $C^{1}$ norms of $\psi, \varphi$ are bounded. The coefficients $d(\psi, \varphi)$, $d_{i j}(\psi, \varphi), c_{i, \gamma}(\psi, \varphi)$, which are polynomials in $\psi, \varphi$ and their derivatives, are also bounded by some constant $C\left(R_{1}\right)$. Note also that $d(\psi, \varphi)$ is bounded from below by a positive constant. Thus, we can choose a sufficiently large $k=k\left(R_{1}\right)$ such that the $K_{i}, K_{i, y}$ above are well defined. Furthermore, maximum principles for elliptic equations give that $K_{i, \gamma}(\psi, \varphi)$ is a positive operator. That is, $K_{i, y}(\psi, \varphi)$ maps $X_{+}^{i}$ into itself.

On the other hand, we can write

$$
\begin{aligned}
& F(u, v)=\left(Q^{v}(f+\gamma u)-P^{v}(g+\gamma v)\right)+k u=[\bar{F}(u, v, \gamma)+k] u, \\
& G(u, v)=\left(P^{u}(g+\gamma v)-Q^{u}(f+\gamma u)\right)+k v=[\bar{G}(u, v, \gamma)+k] v,
\end{aligned}
$$

where $\bar{F}, \bar{G}$ are some continuous functions in $(u, v)$. Clearly, we can choose $k>0$ large enough such that $F(\psi, \varphi), G(\psi, \varphi) \geq 0$ for $(\psi, \varphi) \in U$.

The discussion above allows us to define the maps $T_{\gamma}, T$ from $U$ into $X_{+}$by $T_{\gamma}(\Phi)=\left(K_{1, \gamma}(\Phi)[F(\Phi)], K_{2, \gamma}(\Phi)[G(\Phi)]\right), T(\Phi)=T_{0}(\Phi), \quad$ where $\Phi=(\psi, \varphi)$.

From (3.4), it is clear that $(u, v) \in X$ is a solution for (1.1) if and only if it is a fixed point of $T$ ( or $T_{\gamma}$ ).

3.2. Fixed point index of $T$. We proceed to compute the fixed-point index of the operator $T$. Our goal is to establish the following result.

Theorem 3.1. There exists a $R_{1}>0$ depending on the parameters of (1.1) such that for every $R>R_{1}$, we have

(i) $i\left(T, U_{R}\right)=1$, where $U_{R}:=\left\{x \in X_{+}:\|x\|_{X}<R\right\}$.

(ii) If $u_{0}=v_{0} \equiv 0, X_{+}^{i}$ is invariant under $T$ and $i\left(\left.T\right|_{X_{+}^{i}}, U_{R}^{i}\right)=1$, where $U_{R}^{i}:=$ $U_{R} \cap X_{+}^{i}$.

An immediate consequence of (i) of this theorem is the following solvability result.

Corollary 3.2. Assume that (1.1) satisfies $\left(\mathrm{YYY}^{\prime}\right)$ and that $u_{0}$, $v_{0}$ are nonnegative on $\partial \Omega$. There exists a solution $(u, v)$ to $(1.1)$ with mixed boundary conditions (1.5).

Proof of Theorem 3.1. We first fix a $\gamma>0$ in $[0,1]$ and consider $T_{\gamma}$ (recall that $\left.T=T_{0}\right)$. For each $t \in[0,1]$ and $\Phi=(\psi, \varphi)$, we define the map $T_{\gamma}^{t}: U \rightarrow X_{+}$ 
by $T_{\gamma}^{t}(\Phi)=(u, v)$, with $(u, v)$ being the unique solution to

$$
\left\{\begin{array}{l}
-d(\Phi) \Delta u-d_{11}(\Phi) \nabla u+\left(d_{12}(\Phi)+c_{1, \gamma}(\Phi)+k\right) u=t \tilde{F}(\Phi)+k \psi, \\
-d(\Phi) \Delta v-d_{21}(\Phi) \nabla v+\left(d_{22}(\Phi)+c_{2, \gamma}(\Phi)+k\right) v=t \tilde{G}(\Phi)+k \varphi,
\end{array}\right.
$$

and $\mathcal{B}_{u}^{t}(u)=\mathcal{B}_{v}^{t}(v)=0$ on the boundary. Here,

$$
\begin{aligned}
& \mathcal{B}_{u}^{t}(u)=\left(\frac{\partial u}{\partial v}+r_{1}(x) u\right) \zeta+(1-\zeta)\left(u-t u_{0}\right), \\
& \mathcal{B}_{v}^{t}(v)=\left(\frac{\partial v}{\partial v}+r_{2}(x) v\right) \zeta+(1-\zeta)\left(v-t v_{0}\right) .
\end{aligned}
$$

Let $\vec{u}$ be a fixed point of $T_{\gamma}^{t}$. Multiplying the system $((u, v)=(\psi, \varphi))$ above by the matrix $(1 / d(u, v)) \Theta$ and simplifying the result, we easily see that (compare with (3.4))

$$
\left\{\begin{array}{l}
-\operatorname{div}\left[P^{u}(u, v) \nabla u+P^{v}(u, v) \nabla v\right]=t f(u, v)+(t-1) \gamma u, \\
-\operatorname{div}\left[Q^{u}(u, v) \nabla u+Q^{v}(u, v) \nabla v\right]=t g(u, v)+(t-1) \gamma v .
\end{array}\right.
$$

For $t \in[0,1]$, the right-hand sides of (3.8) are negative if $f(u, v), g(u, v)<$ 0 and $u, v>0$. Theorem 2.2 asserts that, for any nonnegative solution $(u, v)$ of (3.8), $\|u\|_{\infty}$ and $\|v\|_{\infty}$ are bounded by the same bound $K_{\infty}$ for the $L^{\infty}$ norms of solutions of (1.1). Thus, the right-hand sides of (3.8) are bounded by some constant depending on $K_{\infty}$ and the parameters of our original system (1.1), but not on $t, k, \gamma$. Furthermore, Theorem 2.7 asserts that $\|(u, v)\|_{X}$ is bounded by a constant $R_{2}$ depending only on $K_{\infty}$ and the parameters of (1.1) (without $k$ in it). We then redefine $R_{1}$ such that $R_{2}<R_{1}$. Note that the new $R_{1}$ may affect $k$ but not $R_{2}$. Therefore, solutions of (3.8) satisfy $\|(u, v)\|_{X}<R_{1}$.

The argument above shows that $T_{\gamma}^{t}$ has no fixed point on the boundary $\{\vec{u} \in$ $\left.X_{+}:\|\vec{u}\|_{X}=R_{1}\right\}$ of $U$. Hence, we can consider the homotopy $H(t, \bullet)=T_{\gamma}^{t}(\bullet)$. We have

$$
H:[0,1] \times \bar{U} \rightarrow X_{+}
$$

is a compact map and $H(t, \vec{u}) \neq \vec{u}$ for all $(t, \vec{u}) \in[0,1] \times \partial U$.

Applying the homotopy invariance principle (see [1, Theorem 11.1]), we have

$$
i\left(T_{\gamma}, U\right)=i(H(1, \bullet), U)=i(H(0, \bullet), U)=i\left(T_{\gamma}^{0}, U\right) .
$$

Let us study $T_{\gamma}^{0}$. Clearly, a fixed point $(u, v)$ of $T_{\gamma}^{0}$ satisfies

$$
\left\{\begin{array}{l}
-\operatorname{div}\left[P^{u}(u, v) \nabla u+P^{v}(u, v) \nabla v\right]+\gamma u=0, \\
-\operatorname{div}\left[Q^{u}(u, v) \nabla u+Q^{v}(u, v) \nabla v\right]+\gamma v=0,
\end{array}\right.
$$


with homogeneous Robin or Dirichlet boundary conditions on parts of $\partial \Omega$.

We test the equations above for $u, v$ respectively by $u, v$ and sum up the results. As $\gamma>0$, we easily see that $u=v=0$ (as the boundary conditions are homogeneous). Thus, $(0,0)$ is the unique fixed point of $T_{\gamma}^{0}$ in $U$. Note that this is true even for $\gamma=0$ if the mixed boundary condition is considered. This is the only place where we need to introduce the parameter $\gamma>0$ to handle the Neumann boundary condition case.

Let $T_{\gamma}^{0}(\vec{u})=(\Psi, \Phi)$. We have

$$
\left\{\begin{array}{l}
-d(\vec{u}) \Delta \Psi-d_{11}(\vec{u}) \nabla \Psi+\left(d_{12}(\vec{u})+c_{1, \gamma}(\vec{u})+k\right) \Psi=k u, \\
-d(\vec{u}) \Delta \Phi-d_{21}(\vec{u}) \nabla \Phi+\left(d_{22}(\vec{u})+c_{1, \gamma}(\vec{u})+k\right) \Phi=k v .
\end{array}\right.
$$

Linearizing this system at the fixed point $(0,0)$ along the direction $(\psi, \varphi)$, we derive the following system for $(\alpha, \beta)=\left(T_{\gamma}^{0}\right)^{\prime}(0,0)(\psi, \varphi)$ :

$$
\left\{\begin{array}{l}
-d(0,0) \Delta \alpha-d_{11}(0,0) \nabla \alpha+d_{12}(0,0) \alpha+c_{1, \gamma}(0,0) \alpha+k \alpha=k \psi, \\
-d(0,0) \Delta \beta-d_{21}(0,0) \nabla \beta+d_{22}(0,0) \beta+c_{2, \gamma}(0,0) \beta+k \beta=k \varphi .
\end{array}\right.
$$

If $(\psi, \varphi)$ is an eigenvector corresponding to an eigenvalue $\lambda$ for $\left(T_{\gamma}^{0}\right)^{\prime}(0,0)$, then $(\alpha, \beta)=\lambda(\psi, \varphi)$ and the system above gives (with $d_{i j}(0,0)=0$, and $\left.c_{1, \gamma}(0,0)=\gamma \delta_{2}, c_{2, \gamma}(0,0)=\gamma \delta_{1}\right)$

$$
\left\{\begin{array}{l}
-d(0,0) \Delta \psi+\gamma \delta_{2} \psi=\left(\lambda^{-1}-1\right) k \psi \\
-d(0,0) \Delta \varphi+\gamma \delta_{1} \varphi=\left(\lambda^{-1}-1\right) k \varphi
\end{array}\right.
$$

Since $\gamma>0$ and every eigenvalue of $-d(0,0) \Delta+\gamma \delta_{i} I$ is positive, the above gives $\lambda<1$. Using [1, Lemma 13.1], we have $i\left(T_{\gamma}^{0},(0,0)\right)=1$. As we already showed that $(0,0)$ is the only fixed point of $T_{\gamma}^{0}$ on $U$, we have $i\left(T_{\gamma}^{0}, U\right)=$ $i\left(T_{\gamma}^{0},(0,0)\right)=1$.

Thus, from (3.9), $i\left(T_{\gamma}, U\right)=1$. If the mixed boundary condition (1.5) is considered and $\partial \Omega_{0} \neq \varnothing$, then the above has no constant eigenvector and we observe that the argument above still holds for $\gamma=0$. Because $T=T_{0}$, we proved (i) of Theorem 3.1 in this case.

Otherwise, if only the Neumann condition is assumed, we then consider the homotopy $\tilde{H}(\gamma, \bullet)=T_{\gamma}(\bullet), \gamma \in[0,1]$. From the definition of $T_{\gamma}$, we see that the fixed points $(u, v)$ of $\tilde{H}(\gamma, \bullet)$ are exactly the solutions of (1.1); see (3.2). Thus, $\|(u, v)\|_{X}<R_{1}$ and $i(T, U)=i\left(T_{0}, U\right)=i\left(T_{1}, U\right)=1$ by homotopy invariance.

For (ii), since $F(0, \varphi)=G(\psi, 0)=0$ for all $\psi, \varphi \in C^{1}(\Omega)$ and the boundary conditions are now homogeneous, the invariance property follows easily. Finally, a similar (and much simpler) argument as the above establishes the index result for $\left.T\right|_{X_{+}^{i}} \cdot$ 
3.3. Some index results. It is easy to see that if (1.1) satisfies $\left(Y Y Y^{\prime}\right)$ and homogeneous boundary conditions $\left(u_{0}=v_{0}=0\right)$, then $(0,0)$ is one of its solutions. In this case, the conclusion of Corollary 3.2 is not so interesting. To study the existence of positive solutions, we will go further and consider the restrictions of $T$ on the "edges" $X_{+}^{i}, i=1,2$, which are regarded as subsets of $X_{+}$. We now recall some results in [15] that allow us to compute the fixed-point indices of $T$ on the "edges" (or "faces") $X_{+}^{i}$ of $X_{+}$. Let $\beta$ be any subset of $\{1,2\}$. We consider the following sets of fixed points of $T$.

$$
Z_{\beta}:=\left\{\vec{u} \in X_{+}: T(\vec{u})=\vec{u}, u_{i}>0 \text { if } i \in \beta, u_{i}=0 \text { if otherwise }\right\} .
$$

Roughly speaking, $Z_{\beta}$ is the set of fixed points of $T$ on the face $X_{\beta}^{+}=\bigoplus_{i \in \beta} X_{+}^{i}$ and $Z_{\varnothing}=(0,0) . Z=\bigcup_{\beta \neq\{1,2\}} Z_{\beta}$ is the set of all trivial and semitrival fixed points of $T$ on $X_{+}$.

Following [15, Section 3.1], we set

$$
B_{1}(\vec{u}):=K_{1}(\vec{u}) \circ \partial_{u} F(\vec{u}), \quad B_{2}(\vec{u}):=K_{2}(\vec{u}) \circ \partial_{v} G(\vec{u}) .
$$

For sufficiently large $k$ and $\vec{u} \in Z, \partial_{u} F(\vec{u}), \partial_{v} G(\vec{u})$ are positive so that $B_{i}(\vec{u})$ 's are positive operators. We consider the following conditions:

(B) The largest eigenvalue of $B_{i}(0)$ is not equal to 1 for $i=1,2$.

$\left(\mathrm{E}_{i}\right)$ The largest eigenvalue of $B_{i}(0)$ is greater than 1.

(E) $\left(\mathrm{E}_{i}\right)$ holds for all $i \in\{1,2\}$.

Using Theorem 3.1, we have the following result, whose proof is similar to that of [15, Corollary 3.6].

Lemma 3.3. Assume that $(\mathrm{B})$ holds. Iffor some $i \in \beta,\left(\mathrm{E}_{i}\right)$ holds, then $i\left(\left.T\right|_{X_{\beta}^{+}}, 0\right)=$ 0 and $i\left(\left.T\right|_{X_{\beta}^{+}}, Z_{\beta}\right)=1$.

Let $\alpha=\{1,2\} \backslash \beta$. Assuming that $\alpha \neq \varnothing$, we denote $B_{\alpha}=\left(B_{i}\right)_{i \in \alpha}$. If $Z_{\beta}$ is not empty, we consider the following conditions.

$\left(D_{+}^{\beta}\right)$ The largest eigenvalue of $B_{\alpha}(\vec{u})$ is smaller than 1 for all $\vec{u} \in Z_{\beta}$;

$\left(D_{-}^{\beta}\right)$ The largest eigenvalue of $B_{\alpha}(\vec{u})$ is greater than 1 for all $\vec{u} \in Z_{\beta}$.

We say that $\left(\mathrm{D}_{+}\right)$(or $\left.\left(\mathrm{D}_{-}\right)\right)$holds if $\left(\mathrm{D}_{+}^{\beta}\right)$ (or $\left(\mathrm{D}_{-}^{\beta}\right.$ respectively) holds for $\beta=\{1\},\{2\}$.

We then set

$$
\sigma(\beta)=\left\{\begin{array}{ll}
1 & \text { if }\left(\mathrm{D}_{+}^{\beta}\right) \text { holds, } \\
0 & \text { if }\left(\mathrm{D}_{-}^{\beta}\right) \text { holds, }
\end{array} \text { and } \quad i(\beta)=i\left(\left.T\right|_{X_{\beta}^{+}}, Z_{\beta}\right) .\right.
$$

Because $B_{\alpha}(\vec{u})$ is strongly positive, by the Krein-Rutman theorem [1], either $\left(\mathrm{D}_{+}\right)$or $\left(\mathrm{D}_{-}\right)$implies that 1 is not an eigenvalue of $B_{\alpha}(\vec{u})$ corresponding to a positive eigenvector. The following is a restatement of [15, Theorem 3.1]. 
Lemma 3.4. Suppose that either $\left(\mathrm{D}_{+}^{\beta}\right)$ or $\left(\mathrm{D}_{-}^{\beta}\right)$ holds. Then we have $i\left(T, Z_{\beta}\right)=$ $\sigma(\beta) i(\beta)$.

Applying this to our case, we have the following result.

Theorem 3.5. Assume that (E) holds. We have the following:

(i) if $\left(\mathrm{D}_{+}\right)$holds, then

$$
\sum_{\vec{u}_{*} \in Z} i\left(T, \vec{u}_{*}\right)=2
$$

(ii) if $\left(\mathrm{D}_{-}\right)$holds, then

$$
\sum_{\vec{u}_{*} \in Z} i\left(T, \vec{u}_{*}\right)=0 .
$$

Proof. Since (E) implies (B), we can apply Lemma 3.3 here. For $\beta=\{1\}$ or $\{2\}$, we apply Lemma 3.3 to have $i(\beta)=i\left(\left.T\right|_{X_{\beta}^{+}}, Z_{\beta}\right)=1$. Lemma 3.4 then gives $i\left(T, Z_{\beta}\right)=\sigma(\beta) i(\beta)=\sigma(\beta)$. For $\beta=\{1,2\}$, Lemma 3.3 gives $i(T, 0)=$ $i\left(\left.T\right|_{X_{\beta}}, 0\right)=0$. As $Z_{\varnothing}=\{0\}$, we have $i\left(T, Z_{\varnothing}\right)=0$.

Therefore,

$$
\begin{aligned}
\sum_{\vec{u}_{*} \in Z} i\left(T, \vec{u}_{*}\right) & =i\left(T, Z_{\varnothing}\right)+i\left(T, Z_{1}\right)+i\left(T, Z_{2}\right) \\
& =\sigma(\{1\})+\sigma(\{2\})= \begin{cases}2 & \text { if }\left(\mathrm{D}_{+}\right) \text {holds } \\
0 & \text { if }\left(\mathrm{D}_{-}\right) \text {holds }\end{cases}
\end{aligned}
$$

which completes the proof.

The following result is a direct consequence of (i) of Theorem 3.1 and Theorem 3.5 .

Corollary 3.6. Assume that $(E)$ holds, and either $\left(D_{+}\right)$or $\left(D_{-}\right)$holds. System (1.1) has at least one solution $(u, v)$ with $u, v>0$.

\section{The Coexistence Problem}

Corollary 3.6 gives sufficient conditions for the existence of a positive solution to (1.1). In this section, we will present a deeper study on (1.1) satisfying (2.18), which says

$$
\left\{\begin{array} { l } 
{ P ^ { u } = \delta _ { 1 } + a _ { 1 1 } u + a _ { 1 2 } v , } \\
{ P ^ { v } = b _ { 1 1 } u , } \\
{ Q ^ { v } = \delta _ { 2 } + a _ { 2 1 } u + a _ { 2 2 } v , } \\
{ Q ^ { u } = b _ { 2 2 } v , }
\end{array} \quad \text { and } \left\{\begin{array}{l}
f(u, v)=u\left(a_{1}-b_{1} u-c_{1} v\right), \\
g(u, v)=v\left(a_{2}-b_{2} u-c_{2} v\right) .
\end{array}\right.\right.
$$

Our main goal is to determine the range of the parameters in (4.1) such that the hypotheses of Corollary 3.6 are realized. These hypotheses center on the eigenvalue problems of the operators $B_{i}$ 's. 
First of all, we see that for each $\vec{u} \in Z$, the set of all trivial and semitrival fixed points of $T$ on $X_{+}$, the eigenvalue problem $B_{i}(\vec{u}) \varphi=\lambda \varphi$ (see (3.10)) is equivalent to

$$
\left\{\begin{array}{l}
-d(\vec{u}) \Delta \varphi-d_{i 1}(\vec{u}) \nabla \varphi+\left(d_{i 2}(\vec{u})+k\right) \varphi=\lambda^{-1} \hat{F}_{i}(\vec{u}) \varphi, \quad \text { in } \Omega \\
\left(\frac{\partial \varphi}{\partial v}+r_{i}(x) \varphi\right) \zeta+(1-\zeta) \varphi=0, \quad \text { on } \partial \Omega
\end{array}\right.
$$

where $\widehat{F_{1}}(\vec{u})=F_{u}(u, v), \widehat{F_{2}}(\vec{u})=G_{v}(u, v)$.

The conditions $(\mathrm{E}),\left(\mathrm{D}_{+}\right),\left(\mathrm{D}_{-}\right)$then simply read as follows:

$\left(\mathrm{E}_{+}\right)$For each $i=1,2$, the largest eigenvalue of (4.2) is greater than 1 when $\vec{u}=0$.

$\left(D_{+}\right)$For each $i=1,2$ and for any $\vec{u} \in Z_{\{j\}}, i \neq j$, the largest eigenvalue of (4.2) is less than 1.

(D_) For each $i=1,2$ and for any $\vec{u} \in Z_{\{j\}}, i \neq j$, the largest eigenvalue of (4.2) is greater than 1 .

Roughly speaking, (E) says that $(0,0)$ is unstable in the $u, v$ directions; and $\left(\mathrm{D}_{+}\right)$(respectively, $\left(\mathrm{D}_{-}\right)$) requires that a semi-trivial steady state on an edge, e.g. $u$-axis, is stable (respectively, unstable) in its complementary direction, e.g. $v$ direction.

Corollary 3.6 then gives sufficient conditions for coexistence. This general result greatly generalizes [26, Theorem 3.4]. In the sequel, assuming various types of boundary conditions, we will concentrate on the structure (4.1) and find conditions for Corollary 3.6 to be applicable.

4.1. The Robin boundary condition In this section, for the simplicity of stating the results and proofs, we will scale the constants in (1.1) to make $\delta_{1}=$ $\delta_{2}=\delta$ and assume also that $r_{1} \equiv r_{2} \equiv r$ in the boundary conditions.

Setting $u=0$ or $v=0$ in (1.1), we consider the following scalar equations

$$
\left\{\begin{array}{l}
-\nabla\left(\left(\delta+a_{11} u\right) \nabla u\right)=u\left(a_{1}-b_{1} u\right), \\
\frac{\partial u}{\partial n}+r(x) u=0, \quad x \in \partial \Omega
\end{array}\right.
$$

and

$$
\left\{\begin{array}{l}
-\nabla\left(\left(\delta+a_{22} v\right) \nabla v\right)=v\left(a_{2}-c_{2} v\right) \\
\frac{\partial v}{\partial n}+r(x) v=0, \quad x \in \partial \Omega
\end{array}\right.
$$

Let $\mu_{1}$ be the principal eigenvalue of $-\Delta$ on

$$
E=\left\{u \in C^{1}(\Omega): \frac{\partial u}{\partial n}+r(x) u=0\right\} .
$$


By [3, Corollary 3.2], it is now known that the equation for $u$, for example, has a unique positive solution if $\mu_{1}<a_{1} / \delta$. Clearly, $(u, 0)$ and $(0, v)$ constitute the semi-trivial solutions of (1.1).

Our main result in this section is the following.

Theorem 4.1. We suppose $\mu_{1}<\min \left\{a_{1} / \delta, a_{2} / \delta\right\}$. Let $u, v$ be positive solutions to (4.3). Then, system (1.1) with (4.1) has at least one positive solution if either

(i) $b_{22} \geq a_{21}, b_{11} \geq a_{12}$, and for all $x \in \Omega$

$$
\begin{gathered}
\left(a_{1}-b_{1} u(x)\right)\left(\delta+a_{21} u(x)\right)-\left(\delta+a_{11} u(x)\right)\left(a_{2}-b_{2} u(x)\right) \geq 0, \\
\left(a_{2}-c_{2} v(x)\right)\left(\delta+a_{12} v(x)\right)-\left(\delta+a_{22} v(x)\right)\left(a_{1}-c_{1} v(x)\right) \geq 0 .
\end{gathered}
$$

(ii) $b_{22} \leq \min \left\{a_{21}, a_{11}\right\}, b_{11} \leq \min \left\{a_{12}, a_{22}\right\}$, and for all $x \in \Omega$

$$
\begin{gathered}
\left(a_{1}-b_{1} u(x)\right)\left(2 \delta+2 a_{21} u(x)\right)-\left(\delta+a_{11} u(x)\right)\left(a_{2}-b_{2} u(x)\right) \leq 0, \\
\left(a_{2}-c_{2} v(x)\right)\left(2 \delta+2 a_{12} v(x)\right)-\left(\delta+a_{22} v(x)\right)\left(a_{1}-c_{1} v(x)\right) \leq 0 .
\end{gathered}
$$

Proof. Using Lemma 3.6, we need only to verify (E) and either $\left(\mathrm{D}_{+}\right)$or $\left(\mathrm{D}_{-}\right)$.

Condition (E). When $\vec{u}=0,(4.2)$ is equivalent to

$$
\left\{\begin{array}{l}
-\delta^{2} \Delta \varphi+k \varphi=\lambda^{-1}\left(a_{i} \delta+k\right) \varphi, \\
\frac{\partial \varphi}{\partial v}+r(x) \varphi=0 .
\end{array}\right.
$$

From the condition $\mu_{1}<\min _{i}\left\{a_{i} / \delta\right\}$ we have $\lambda:=\left(\delta a_{i}+k\right) /\left(\mu_{1} \delta^{2}+k\right)>1$ is an eigenvalue of (4.4) corresponding to $e_{1}$ (the eigenvector of $-\Delta$ associated with the eigenvalue $\mu_{1}$ ). So (E) holds.

Conditions $\left(\mathrm{D}_{+}\right)$and $\left(\mathrm{D}_{-}\right)$. Consider $i=2$ and $\vec{u}=(u, 0) \in Z_{1}$, the set of semitrivial solutions in the $u$-axis. Let $\lambda$ be the largest eigenvalue of $B_{2}(\vec{u})$. It is well known (see [1]) that there exists $\varphi>0$ such that $B_{2}(\vec{u}) \varphi=\lambda \varphi$. From (4.2) and (3.5), we have the equation

$$
\begin{aligned}
-P^{u} Q^{v} \Delta \varphi-\left(Q_{v}^{u}+Q_{u}^{v}\right) P^{u} \nabla u \nabla \varphi+\left(P_{u}^{u} Q_{v}^{u}|\nabla u|^{2}+k\right) \varphi & \\
& =\lambda^{-1}\left(P^{u} g_{v}-Q_{v}^{u} f+k\right) \varphi .
\end{aligned}
$$

Here and throughout this section, the functions $P^{u}, P^{v}, Q^{u}, Q^{v}, f, g$ and their derivatives are evaluated at $\vec{u}=(u, 0)$.

The equation above is equivalent to

$$
\begin{aligned}
-\nabla\left[P^{u} Q^{v} \nabla \varphi\right]-\left(Q_{v}^{u} P^{u}-Q^{v} P_{u}^{u}\right) \nabla u \nabla \varphi+ & \left(P_{u}^{u} Q_{v}^{u}|\nabla u|^{2}+k\right) \varphi \\
& =\lambda^{-1}\left(P^{u} g_{v}-Q_{v}^{u} f+k\right) \varphi .
\end{aligned}
$$


Testing this equation with $\Psi=\Psi(u)>0$, which will be determined later, and taking integration by parts, we have

$$
\begin{aligned}
\int_{\partial \Omega} P^{u} Q^{v} \Psi r & (x) \varphi \mathrm{d} \sigma+\int_{\Omega}\left[P^{u} Q^{v} \Psi_{u}-\left(Q_{v}^{u} P^{u}-Q^{v} P_{u}^{u}\right) \Psi\right] \nabla \varphi \nabla u \mathrm{~d} x \\
& +\int_{\Omega}\left(P_{u}^{u} Q_{v}^{u}|\nabla u|^{2}+k\right) \Psi \varphi \mathrm{d} x=\lambda^{-1} \int_{\Omega}\left(P^{u} g_{v}-Q_{v}^{u} f+k\right) \Psi \varphi \mathrm{d} x .
\end{aligned}
$$

Let $N:=\int_{\Omega}\left(P^{u} g_{v}-Q_{v}^{u} f+k\right) \Psi \varphi \mathrm{d} x$. A little rearrangement of the above shows that $\left(\lambda^{-1}-1\right) N$ is equal to

$$
\begin{array}{r}
\int_{\partial \Omega} P^{u} Q^{v} \Psi r(x) \varphi \mathrm{d} \sigma+\int_{\Omega}\left[P^{u} Q^{v} \Psi_{u}-\left(Q_{v}^{u} P^{u}-Q^{v} P_{u}^{u}\right) \Psi\right] \nabla \varphi \nabla u \mathrm{~d} x \\
+\int_{\Omega} P_{u}^{u} Q_{v}^{u} \Psi \varphi|\nabla u|^{2} \mathrm{~d} x-\int_{\Omega}\left(P^{u} g_{v}-Q_{v}^{u} f\right) \Psi \varphi \mathrm{d} x .
\end{array}
$$

By choosing $k$ sufficiently large, we have $\left(P^{u} g_{v}-Q_{v}^{u} f+k\right)>0$ and $N>0$. Hence, the sign of $\left(\lambda^{-1}-1\right)$ is that of the quantity above, which will be our focus below. Our first task is to get rid of the term involving $\nabla u \nabla \varphi$. Since $(u, 0)$ is a fixed point of $T$, it satisfies system (1.1). From the first equation of (1.1), we have

$$
-\operatorname{div}\left[P^{u}(u, 0) \nabla u\right]=f(u, 0) .
$$

Test this with $\bar{P} \varphi$, in which

$$
\bar{P}=Q^{v} \Psi_{u}-Q_{v}^{u} \Psi+\frac{Q^{v} P_{u}^{u} \Psi}{P^{u}},
$$

to derive

$$
\int_{\Omega} P^{u} \bar{P} \nabla u \nabla \varphi \mathrm{d} x=-\int_{\Omega} P^{u} \bar{P}_{u} \varphi|\nabla u|^{2} \mathrm{~d} x+\int_{\Omega} f \bar{P} \varphi \mathrm{d} x-\int_{\partial \Omega} P^{u} \bar{P} r u \varphi \mathrm{d} \sigma .
$$

The left integral of the above is nothing but the second term in (4.5). Therefore, by substituting this equality into (4.5) and then grouping the result, we obtain

$$
\left(\lambda^{-1}-1\right) N=\int_{\partial \Omega} I \partial r \varphi \mathrm{d} \sigma+\int_{\Omega} I_{\Omega} \varphi|\nabla u|^{2} \mathrm{~d} x+\int_{\Omega} I_{R} \varphi \mathrm{d} x,
$$

where

$$
\begin{aligned}
& I_{\partial}=\left(P^{u} Q^{v} \Psi-P^{u} \bar{P} u\right), \\
& I_{\Omega}=\left(P_{u}^{u} Q_{v}^{u} \Psi-P^{u} \bar{P}_{u}\right), \\
& I_{R}=\left(f \bar{P}-P^{u} g_{v} \Psi+Q_{v}^{u} f \Psi\right) .
\end{aligned}
$$


We further simplify these terms by using the definition of $\bar{P},(4.1)$, and

$$
\bar{P}_{u}=Q_{u}^{v} \Psi_{u}+Q^{v} \Psi_{u u}-Q_{v}^{u} \Psi_{u}+\frac{Q_{u}^{v} P_{u}^{u} \Psi+Q^{v} P_{u}^{u} \Psi_{u}}{P^{u}}-\frac{Q^{v} \Psi\left(P_{u}^{u}\right)^{2}}{\left(P^{u}\right)^{2}} .
$$

We get

$$
\begin{aligned}
& I_{\partial}=u P^{u}\left(Q_{v}^{u} \Psi-Q^{v} \Psi_{u}\right)+Q^{v} \Psi\left(P^{u}-P_{u}^{u} u\right), \\
& I_{\Omega}=\left(Q_{v}^{u}-Q_{u}^{v}\right)\left(P_{u}^{u} \Psi+P^{u} \Psi_{u}\right)+Q^{v}\left[\frac{\Psi\left(P_{u}^{u}\right)^{2}}{P^{u}}-P^{u} \Psi_{u u}-P_{u}^{u} \Psi_{u}\right], \\
& I_{R}=\frac{f Q^{v}}{P^{u}}\left(P^{u} \Psi_{u}+P_{u}^{u} \Psi\right)-P^{u} g_{v} \Psi .
\end{aligned}
$$

Since $\varphi>0$ and $N>0$, as we mentioned earlier, (4.7) shows that $\lambda>1$ (respectively, $\lambda<1$ ) if $I_{\partial}, I_{\Omega}$ and $I_{R}$ are negative (respectively, positive) altogether. Let us consider these two cases.

The case $\left(\mathrm{D}_{+}\right)(\lambda<1)$. In this case, we shall choose $\Psi=u / P^{u}$. Noting that

$$
\Psi_{u}=\frac{1}{P^{u}}-P_{u}^{u} \frac{u}{\left(P^{u}\right)^{2}}, \quad \Psi_{u u}=-2 \frac{P_{u}^{u}}{\left(P^{u}\right)^{2}}+2\left(P_{u}^{u}\right)^{2} \frac{u}{\left(P^{u}\right)^{3}},
$$

we compute and get

$$
\begin{aligned}
& I_{\partial}=Q_{v}^{u} u^{2}, \\
& I_{\Omega}=\left(Q_{v}^{u}-Q_{u}^{v}\right)+\frac{P_{u}^{u} Q^{v}}{P^{u}}, \\
& I_{R}=\frac{1}{P^{u}}\left(Q^{v} f-P^{u} g_{v} u\right) .
\end{aligned}
$$

Therefore, these terms will be positive if $Q_{v}^{u}-Q_{u}^{v} \geq 0$ and $Q^{v} f-P^{u} g_{v} u \geq 0$, or

$b_{22} \geq a_{21} \quad$ and $\quad\left(a_{1}-b_{1} u\right)\left(\delta+a_{21} u\right)-\left(\delta+a_{11} u\right)\left(a_{2}-b_{2} u\right) \geq 0$.

The case $\left(\mathrm{D}_{-}\right)(\lambda>1)$. We now choose $\Psi=u$ and find that

$$
\begin{aligned}
& I_{\partial}=\left(Q_{v}^{u}-Q_{u}^{v}\right) P_{u}^{u} u^{3}+\left(Q_{v}^{u}-P_{u}^{u}\right) \delta u^{2}, \\
& I_{\Omega}=\left(Q_{v}^{u}-Q_{u}^{v}\right)\left(P_{u}^{u} u+P^{u}\right)-\frac{\delta Q^{v} P_{u}^{u}}{P^{u}}, \\
& I_{R}=\frac{f Q^{v}}{P^{u}}\left(P^{u}+P_{u}^{u} u\right)-P^{u} g_{v} u \leq 2 f Q^{v}-P^{u} g_{v} u .
\end{aligned}
$$


They are nonpositive if $Q_{v}^{u}-Q_{u}^{v} \leq 0, Q_{v}^{u}-P_{u}^{u} \leq 0$ and $2 f Q^{v}-P^{u} g_{v} u \leq 0$, or

$b_{22} \leq \min \left\{a_{21}, a_{11}\right\} \quad$ and $\quad\left(a_{1}-b_{1} u\right)\left(2 \delta+2 a_{21} u\right)-\left(\delta+a_{11} u\right)\left(a_{2}-b_{2} u\right) \leq 0$.

By symmetry, we require the same conditions for the semitrivial solution $(0, v)$ in each case. It is clear that those are the stated conditions in the theorem. We finish the proof for Theorem 4.1.

Remark 4.2. It is not too difficult to substantiate the conditions (i) of Theorem 4.1. For example, large cross diffusions will be enough for (i) in the strong competition case where we are given that $b_{1} / b_{2}<a_{1} / a_{2}<c_{1} / c_{2}$. In fact, let us consider the first inequality in (i).

$$
\left(a_{1}-b_{1} u(x)\right)\left(\delta+a_{21} u(x)\right)-\left(\delta+a_{11} u(x)\right)\left(a_{2}-b_{2} u(x)\right) \geq 0 .
$$

It is well known that $u$, the positive solution to (4.3), satisfies $0<\eta \leq u(x) \leq$ $a_{1} / b_{1}$ for all $x \in \Omega$. If $u(x) \geq a_{2} / b_{2}$, then the inequality above is trivial. Otherwise, $\left(a_{1}-b_{1} u(x)\right) \geq\left(a_{1}-b_{1} a_{2} / b_{2}\right)>0$ and we can choose $a_{21}=C\left(a_{i}, b_{i}, \eta\right)$ sufficiently large so that the inequality above holds. This is possible because $\eta$, the positive lower bound for $u$, does not depend on $a_{21}$. Similarly, large $a_{12}$ will give the second inequality in (i). Thus, strong cross diffusions will be sufficient for this case. Nevertheless, the verification of the condition (ii) seems to be more subtle as we may need to have a better understanding on the minimum values of the solutions $u, v$ of (4.3) on $\Omega$. The Dirichlet boundary condition case can be treated similarly but also requires more technicalities concerning the semitrivial solutions. Results such as those just outlined will appear in a forthcoming paper.

4.2. The Neumann boundary condition. In this section we consider (1.1) with (4.1) and Neumann boundary conditions (see, however, Remark 4.17 for possible generalizations). We will mainly focus on the so called "weak" or "strong" competition cases:

(S) (Strong competition: ) $b_{1} / b_{2}<a_{1} / a_{2}<c_{1} / c_{2}$.

(W) (Weak competition: ) $b_{1} / b_{2}>a_{1} / a_{2}>c_{1} / c_{2}$.

The existence of a positive solution for $(1.1)$ in these cases is trivial since $\left(u_{*}, v_{*}\right)=$ $\left(\left(a_{1} c_{2}-a_{2} c_{1}\right) /\left(b_{1} c_{2}-b_{2} c_{1}\right),\left(b_{1} a_{2}-b_{2} a_{1}\right) /\left(b_{1} c_{2}-b_{2} c_{1}\right)\right)$ is a positive solution for the problem. Of course, this constant solution is not interesting both mathematically and biologically. The purpose of this section is to find the conditions on the parameters of (1.1) that guarantee the existence of a positive nonconstant solution (or, in biological terms, pattern formation). Throughout this section, we will assume the condition (B.1) so that the index results of Theorem 3.1 hold.

We denote by $\mu_{i}, i=0,1, \ldots$, the eigenvalues of $-\Delta$ with Neumann's boundary condition. Note that $\mu_{0}=0$. We also denote by $m\left(\mu_{i}\right)$ the algebraic multiplicity of $\mu_{i}$.

Concerning the fixed point indices of the trivial and semitrivial solutions, we have the following result. 

$T$.

Proposition 4.3. Let $Z$ be the set of all the trivial and semitrivial fixed points of

(i) If (S) holds, then $\sum_{\vec{u} \in Z} i(T, \vec{u})=2$.

(ii) If (W) holds, then $\sum_{\vec{u} \in Z} i(T, \vec{u})=0$.

Proof. (i) We will verify the conditions (E), $\left(\mathrm{D}_{+}\right)$. When $\vec{u}=0$, (4.2) (see also (4.4)) reads

$$
-\delta_{i}^{2} \Delta \varphi+k \varphi=\lambda^{-1}\left(a_{i} \delta_{i}+k\right) \varphi, \quad \frac{\partial \varphi}{\partial v}=0 .
$$

Clearly, $\lambda=\left(a_{i} \delta_{i}+k\right) / k>1$ is an eigenvalue of this system with respect to $\varphi=1>0$. Hence, (E) holds for $i=1,2$.

Next, the semitrivial fixed points of $T$ are $(u, 0)=\left(a_{1} / b_{1}, 0\right)$ and $(0, v)=$ $\left(0, a_{2} / c_{2}\right)$. Let us look at $(u, 0)$. The eigenvalue problem for $B_{2}(u, 0)$ in $(4.2)$ is $-d(u, 0) \Delta \varphi-d_{21}(u, 0) \nabla \varphi+\left(d_{22}(u, 0)+k\right) \varphi=\lambda^{-1} \frac{\partial}{\partial v} G(u, 0) \varphi, \quad \frac{\partial \varphi}{\partial v}=0$, or

$$
-d(u, 0) \Delta \varphi+k \varphi=\lambda^{-1}\left[\left(\delta_{1}+a_{11} u\right)\left(a_{2}-b_{2} u\right)+k\right] \varphi .
$$

Thanks to (S), $a_{2}-b_{2} u<0\left(u=a_{1} / b_{1}\right)$. So, if $k$ is sufficiently large we see that $\varphi=1$ is the positive eigenfunction corresponding to the eigenvalue

$$
\lambda=\frac{\left(\delta_{1}+a_{11} u\right)\left(a_{2}-b_{2} u\right)+k}{k}<1 .
$$

The same argument applies to the semitrivial solution $(0, v)$. Therefore, $\left(\mathrm{D}_{+}\right)$ holds. Using (i) of Theorem 3.5, we proved (i). Similar computations establish (ii) by verifying ( $\left.\mathrm{D}_{-}\right)$.

One of the key ingredients of our analysis in this section is to compute the index $i\left(T, \vec{u}_{*}\right)$ at the positive (constant) fixed point of $T$. The following result provides the formula.

Theorem 4.4. Suppose that the matrix

$$
\mathcal{M}=\left(\begin{array}{ll}
F_{u}\left(\vec{u}_{*}\right) & F_{v}\left(\vec{u}_{*}\right) \\
G_{u}\left(\vec{u}_{*}\right) & G_{v}\left(\vec{u}_{*}\right)
\end{array}\right)
$$

has two distinct eigenvalues. Let $\mathcal{N}_{i}:=I-\left(1 /\left(d\left(\overrightarrow{\mathcal{u}}_{*}\right) \mu_{i}+k\right)\right) \mathcal{M}$, where $I$ is the $2 \times 2$ identity matrix. Suppose that the matrices $\mathcal{N}_{i}$ are non-singular for all $i \geq 0$. Then

$$
i\left(T, \vec{u}_{*}\right)=(-1)^{k^{*}},
$$

where

$$
k^{*}=\sum_{\operatorname{det} \mathcal{N}_{i}<0} m\left(\mu_{i}\right) .
$$


To proceed, we first compute $T^{\prime}\left(\vec{u}_{*}\right)(\psi, \varphi)$, the directional derivative of $T$ at $\vec{u}_{*}$ along the direction $\Phi=(\psi, \varphi)$. Let $(U, V)=T^{\prime}\left(\vec{u}_{*}\right)(\psi, \varphi)$. Linearizing the system

$$
\left\{\begin{array}{l}
d(\vec{u}) \Delta u-d_{11}(\vec{u}) \nabla u+\left(d_{12}(\vec{u})+k\right) u=F(\vec{u}), \\
-d(\vec{u}) \Delta v-d_{21}(\vec{u}) \nabla v+\left(d_{22}(\vec{u})+k\right) v=G(\vec{u}),
\end{array}\right.
$$

we easily get

$$
\left\{\begin{array}{l}
-d\left(\vec{u}_{*}\right) \Delta U-d_{11}\left(\vec{u}_{*}\right) \nabla U+\left(d_{12}\left(\vec{u}_{*}\right)+k\right) U=\hat{F} \\
-d\left(\vec{u}_{*}\right) \Delta V-d_{21}\left(\vec{u}_{*}\right) \nabla V+\left(d_{22}\left(\vec{u}_{*}\right)+k\right) V=\hat{G} .
\end{array}\right.
$$

Here,

$$
\begin{aligned}
\hat{F}=- & {\left[-\nabla_{\vec{u}} d\left(\vec{u}_{*}\right) \cdot \Phi \Delta u_{*}-\nabla_{\vec{u}} d_{11}\left(\vec{u}_{*}\right) \cdot \Phi \nabla u_{*}+\nabla_{\vec{u}} d_{12}\left(\vec{u}_{*}\right) \cdot \Phi u_{*}\right] } \\
& +\nabla_{\vec{u}} F\left(\vec{u}_{*}\right) \cdot \Phi, \\
\hat{G}=- & \left.-\nabla_{\vec{u}} d\left(\vec{u}_{*}\right) \cdot \Phi \Delta v_{*}-\nabla_{\vec{u}} d_{21}\left(\vec{u}_{*}\right) \cdot \Phi \nabla v_{*}+\nabla_{\vec{u}} d_{22}\left(\vec{u}_{*}\right) \cdot \Phi v_{*}\right] \\
& +\nabla_{\vec{u}} G\left(\vec{u}_{*}\right) \cdot \Phi .
\end{aligned}
$$

Because $\vec{u}_{*}$ is constant, the above system reduces to

$$
\left\{\begin{array}{l}
-d\left(\vec{u}_{*}\right) \Delta U+k U=\nabla_{\vec{u}} F\left(\vec{u}_{*}\right) \cdot(\psi, \varphi), \\
-d\left(\vec{u}_{*}\right) \Delta V+k V=\nabla_{\vec{u}} G\left(\vec{u}_{*}\right) \cdot(\psi, \varphi) .
\end{array}\right.
$$

Recall that $(U, V)=T^{\prime}\left(\vec{u}_{*}\right)(\psi, \varphi)$. The above says that $T^{\prime}\left(\vec{u}_{*}\right)$ is a compact linear map from $X$ into itself with

$$
T^{\prime}\left(\vec{u}_{*}\right) \Phi=\left[-d\left(\vec{u}_{*}\right) \operatorname{diag}(\Delta, \Delta)+k I\right]^{-1} \mathcal{M} \Phi, \quad \Phi=(\psi, \varphi)^{T} .
$$

Here, $-d\left(\vec{u}_{*}\right) \operatorname{diag}(\Delta, \Delta)+k I$ is armed with the Neumann boundary condition. We now apply the Leray index theorem to compute the fixed point index of $T$ at $\vec{u}_{*}$.

Proposition 4.5 ([1, Theorem 11.4]). Assume that 1 is not an eigenvalue of $T^{\prime}\left(\vec{u}_{*}\right)$. Then

$$
i\left(T,\left(\vec{u}_{*}\right)\right)=(-1)^{m}, \quad m=\sum_{\lambda>1} n_{\lambda} .
$$

Here $n_{\lambda}$ is the algebraic multiplicity of the eigenvalue $\lambda$ of $T^{\prime}\left(\vec{u}_{*}\right)$. That is

$$
n_{\lambda}:=\operatorname{dim}\left[\bigcup_{p=1}^{\infty} \operatorname{ker}\left(\lambda I-T^{\prime}\left(\vec{u}_{*}\right)\right)^{p}\right] .
$$


Let us consider the Hilbert space $\mathcal{E}=H^{1}(\Omega)$. Let $\mathcal{T}$ be the extension of $T^{\prime}\left(\vec{u}_{*}\right)$ on $\mathcal{X}=\mathcal{E} \times \mathcal{E}$. From the regularity theory of Laplacian equations, we see that the eigenvectors of $\mathcal{T}$ in $\mathcal{X}$ are smooth so that generalized eigenspaces $\operatorname{ker}\left(\lambda I-T^{\prime}\left(\vec{u}_{*}\right)\right)^{p}$ coincide with $\operatorname{ker}(\lambda I-\mathcal{T})^{p}$. Thus we will study the eigenspaces of $\mathcal{T}$ on the Hilbert space $\chi$. The following result, which is a little more general than what we need, will serve our purpose.

Lemma 4.6. Suppose that $\mathcal{M}$ is a $n \times n$ matrix. Let $\mathcal{E}$ be a Hilbert space and $L: \mathcal{E} \longrightarrow \mathcal{E}$ be a self adjoint compact operator. We define

$$
\begin{aligned}
\mathcal{T}: \mathcal{E}^{n} & \rightarrow \mathcal{E}^{n}, \\
\mathcal{T} & =\mathcal{L} \mathcal{M},
\end{aligned}
$$

where $\mathcal{L}=L \times L \times \cdots \times L: \mathcal{E}^{n} \longrightarrow \mathcal{F}^{n}$ and $\mathcal{M}$ is regarded as a linear map $\mathcal{E}^{n}$ into itself. Let $\left\{\mu_{i}\right\}_{i=1}^{\infty},\left\{e_{i}\right\}_{i=1}^{\infty}$ (respectively, $\left.\left\{\gamma_{i}\right\}_{i=1}^{n},\left\{u_{i}\right\}_{i=1}^{n}\right)$ be the sequences of distinct eigenvalues and eigenvectors of $L$ (respectively, $\mathcal{M})$. We then have

(i) $\operatorname{ker}(\lambda I-\mathcal{T})=\operatorname{span}\left\{u_{i} e_{j}: \mathcal{M} u_{i}=\gamma u_{i}\right.$, and $L e_{j}=\mu e_{j}$, with $\left.\lambda=\gamma \mu\right\}$.

(ii) If $n_{\gamma_{i}}=\chi_{\gamma_{i}}$ for all eigenvalues $\gamma_{i}$ of $\mathcal{M}$ (here, $\chi_{\gamma}$ is the geometric multiplicity of the eigenvalue $\gamma$ ), then we have $n_{\lambda}=\chi_{\lambda}$ for every eigenvalue $\lambda$ of $\mathcal{T}$. Moreover,

$$
\chi_{\lambda}=\operatorname{dim}[\operatorname{ker}(\lambda I-\mathcal{T})]=\sum n_{\mu_{i}} n_{\gamma_{j}}
$$

where the summation is taken over all $\gamma_{j}, \mu_{i}$ such that $\lambda=\gamma_{j} \mu_{i}$.

This result may be known, but we could not find the reference. Hence, we give a sketch of its proof.

Proof. Let $x \neq 0$ be in $\operatorname{ker}(\lambda I-\mathcal{T})$. Since $\left\{e_{i}\right\}_{i=1}^{\infty}$ forms a basis for $\mathcal{E}$, we can write $x=\sum \alpha_{i} e_{i}$ for $\alpha_{i} \in \mathbb{R}^{n}$. From $\mathcal{T} x=\lambda x$, we have $\sum M \alpha_{i} \mu_{i} e_{j}=\lambda \sum \alpha_{i} e_{j}$ or $\sum\left(\mu_{i} M \alpha_{i}-\lambda \alpha_{i}\right) e_{j}=0$. This implies that $\mu_{i} M \alpha_{i}=\lambda \alpha_{i}$ for all $i$. Since $x \neq 0$, there is an $i$ such that $\alpha_{i}$ is an eigenvector of $\mathcal{M}$ with eigenvalue $\lambda / \mu_{i}$. Thus, $x$ is a (finite) linear combination of $u_{i} e_{j}$. Furthermore, since $\left\{u_{i}\right\}$ and $\left\{e_{i}\right\}$ are linear independent sets, we easily see that $\left\{u_{i} e_{j}\right\}$ is also a linear independent set. This shows that the set $\left\{u_{i} e_{j}: M u_{i}=\gamma u_{i}\right.$, and $L e_{j}=\mu e_{j}$, with $\left.\lambda=\gamma \mu\right\}$ forms a basis for $\operatorname{ker}(\lambda I-\mathcal{T})$. This proves (i).

We now recall [30, Proposition 8.18] which states that

$$
n_{\lambda}=x_{\lambda} \Longleftrightarrow \operatorname{det}\left(\left\langle x_{i}^{*}, x_{j}\right\rangle\right) \neq 0,
$$

where $\left\{x_{1}, \ldots, x_{n}\right\}$ and $\left\{x_{1}^{*}, \ldots, x_{n}^{*}\right\}$ are the bases of $N=\operatorname{ker}(\lambda I-\mathcal{T})$ and $N^{*}=\operatorname{ker}\left(\lambda I-\mathcal{T}^{*}\right)$, respectively. Since $\mathcal{T}^{*}=M^{*} \mathcal{L}^{*}=M^{*} \mathcal{L}$, similar argument as above shows that

$$
N^{*}=\operatorname{span}\left\{u_{i}^{*} e_{j}: M^{*} u_{i}^{*}=\gamma u_{i}^{*}, \text { and } L e_{j}=\mu e_{j}, \text { with } \lambda=\gamma \mu\right\} .
$$


We can assume that $\left\{e_{j}\right\}$ is an orthonormal basis for $\mathcal{E}$. Note that $\left\langle u_{\ell}^{*} e_{i}, u_{k} e_{j}\right\rangle=$ $\left\langle u_{\ell}^{*}, u_{k}\right\rangle\left\langle e_{i}, e_{j}\right\rangle$. It is easy to see that we can rearrange the bases of $N, N^{*}$ such that the matrix $\left(\left\langle x_{i}^{*}, x_{j}\right\rangle\right)$ is a block diagonal matrix whose diagonal blocks are just the matrices $\left(\left\langle u_{\ell}^{*}, u_{m}\right\rangle\right)$, which are formed from the eigenvectors of $M$, $M^{*}$ corresponding the same eigenvalue $\lambda / \mu_{i}$. Apply [30, Proposition 8.18] to the matrix $M$ to see that the determinants of these matrices are nonzero, so is $\operatorname{det}\left(\left\langle x_{i}^{*}, x_{j}\right\rangle\right)$. This establishes (ii). Since $L$ is self adjoint, $n_{\mu_{i}}=\chi_{\mu_{i}}$ (see [30]). (4.9) then follows from (i).

We are now ready to give the proof of Theorem 4.4.

Proof. We apply Lemma 4.6 to $L=\left(-d\left(\vec{u}_{*}\right) \Delta+k I\right)^{-1}$ and $\mathcal{M}$ defined in the theorem. The eigenvalues of the self adjoint operator $L$ are $1 /\left(d\left(\vec{u}_{*}\right) \mu_{i}+k\right)$ with the multiplicities $m\left(\mu_{i}\right)$. Supposing that $\mathcal{M}$ has two different eigenvalues $\gamma_{1}<\gamma_{2}$, we have $n_{\gamma_{i}}=\chi_{\gamma_{i}}=1$ for $i=1$, 2. From (4.9), we have

$$
n_{\lambda}=\sum_{\lambda=\gamma_{1} /\left(d\left(\vec{u}_{*}\right) \mu_{i}+k\right)} m\left(\mu_{i}\right)+\sum_{\lambda=\gamma_{2} /\left(d\left(\vec{u}_{*}\right) \mu_{i}+k\right)} m\left(\mu_{i}\right) .
$$

Therefore,

$$
\begin{aligned}
m=\sum_{\lambda>1} n_{\lambda} & =\sum_{\lambda_{i}^{1}>1} m\left(\mu_{i}\right)+\sum_{\lambda_{i}^{2}>1} m\left(\mu_{i}\right), \\
\lambda_{i}^{1} & =\frac{\gamma_{1}}{d\left(\vec{u}_{*}\right) \mu_{i}+k}, \\
\lambda_{i}^{2} & =\frac{\gamma_{2}}{d\left(\vec{u}_{*}\right) \mu_{i}+k} .
\end{aligned}
$$

Let $\mathcal{I}=\left\{i: \lambda_{i}^{1}<1<\lambda_{i}^{2}\right\}$. We then have

$$
\begin{aligned}
m & \equiv\left(\sum_{i \in \mathcal{I}} m\left(\mu_{i}\right)\right)(\bmod 2) \\
& \equiv\left(\sum_{\left(1-\lambda_{i}^{1}\right)\left(1-\lambda_{i}^{2}\right)<0} m\left(\mu_{i}\right)\right)(\bmod 2) .
\end{aligned}
$$

Noting that $\left(1-\lambda_{i}^{1}\right)$ and $\left(1-\lambda_{i}^{2}\right)$ are the eigenvalues of the space $\mathcal{N}_{i}=$ $I-\left(1 /\left(d\left(\vec{u}_{*}\right) \mu_{i}+k\right)\right) \mathcal{M}$, we have $\mathcal{I}=\left\{i: \operatorname{det} \mathcal{N}_{i}<0\right\}$. Therefore,

$$
m \equiv\left(\sum_{\operatorname{det} \mathcal{N}_{i}<0} m\left(\mu_{i}\right)\right)(\bmod 2) \equiv k^{*}(\bmod 2),
$$

which, together with Proposition 4.5, gives us the proof of the theorem. 
We now combine the results of Theorem 4.4 and Proposition 4.3 to find sufficient conditions for the existence of a positive nonconstant solution to system (1.1).

To apply Theorem 4.4, let us consider $\mathcal{N}_{i}$. We have

$$
\begin{aligned}
\mathcal{N}_{i} & =\frac{1}{d\left(\vec{u}_{*}\right) \mu_{i}+k}\left[\left(\begin{array}{cc}
Q^{v} & -P^{v} \\
-Q^{u} & P^{u}
\end{array}\right)\left(\begin{array}{ll}
b_{1} u_{*} & c_{1} u_{*} \\
b_{2} v_{*} & c_{2} v_{*}
\end{array}\right)+\mu_{i} d\left(\vec{u}_{*}\right) I\right] \\
& =\frac{1}{d\left(\vec{u}_{*}\right) \mu_{i}+k}\left(\begin{array}{cc}
Q^{v} & -P^{v} \\
-Q^{u} & P^{u}
\end{array}\right)\left[\left(\begin{array}{ll}
b_{1} u_{*} & c_{1} u_{*} \\
b_{2} v_{*} & c_{2} v_{*}
\end{array}\right)+\mu_{i}\left(\begin{array}{cc}
P^{u} & P^{v} \\
Q^{u} & Q^{v}
\end{array}\right)\right] .
\end{aligned}
$$

Since the determinant of the first matrix is positive, we have

$$
\begin{aligned}
& \operatorname{det}\left(\mathcal{N}_{i}\right)<0 \\
& \quad \Leftrightarrow D\left(\mu_{i}\right):=\operatorname{det}\left[\left(\begin{array}{ll}
b_{1} u_{*} & c_{1} u_{*} \\
b_{2} v_{*} & c_{2} v_{*}
\end{array}\right)+\mu_{i}\left(\begin{array}{ll}
P^{u} & P^{v} \\
Q^{u} & Q^{v}
\end{array}\right)\right]<0 .
\end{aligned}
$$

A simple calculation gives

$$
D(\mu)=d\left(u_{*}, v_{*}\right) \mu^{2}+d_{1}\left(u_{*}, v_{*}\right) \mu+d_{2}\left(u_{*}, v_{*}\right),
$$

where

$$
\begin{aligned}
d\left(\vec{u}_{*}\right) & =P^{u}\left(\vec{u}_{*}\right) Q^{v}\left(\vec{u}_{*}\right)-P^{v}\left(\vec{u}_{*}\right) Q^{u}\left(\vec{u}_{*}\right), \\
d_{1}\left(\vec{u}_{*}\right) & =P^{u}\left(\vec{u}_{*}\right) c_{2} v_{*}-Q^{u}\left(\vec{u}_{*}\right) c_{1} u_{*}+Q^{v}\left(\vec{u}_{*}\right) b_{1} u_{*}-P^{v}\left(\vec{u}_{*}\right) b_{2} v_{*}, \\
d_{2}\left(\vec{u}_{*}\right) & =\left(b_{1} c_{2}-b_{2} c_{1}\right) u_{*} v_{*} .
\end{aligned}
$$

Let us first assume that $D(\mu)$ can attain negative values on $\mathbb{R}$ (see Remark 4.8 below). We have the following result.

Theorem 4.7. Suppose that $D\left(\mu_{i}\right) \neq 0$ for all $i \geq 0$ and the set $\mathcal{O}:=\{i \geq 1$ : $\left.D\left(\mu_{i}\right)<0\right\}$ is nonempty. Let $i_{0}=\min \mathcal{O}$ and $i_{1}=\max \mathcal{O}$. Assume also that

$$
\sum_{i=i_{0}}^{i_{1}} m\left(\mu_{i}\right) \text { is odd, }
$$

and either (S) or (W); then system (1.1) with (4.1) has at least one positive nonconstant solution.

Proof. We first note that the condition $\mathcal{O} \neq \varnothing$ implies that there exists an $i$ such that $\mathcal{N}_{i}$ has two distinct eigenvalues $\lambda_{i}^{1}<0<\lambda_{i}^{2}$. So, the matrix $\mathcal{M}$ also has two distinct eigenvalues. Moreover, since $D\left(\mu_{i}\right) \neq 0, \mathcal{N}_{i}$ is non-singular for all $i$. Therefore, all the conditions of Proposition 4.3 are satisfied. Also note that, since 
$D(\mu)$ is a quadratic function of $\mu$ with $d\left(\vec{u}_{*}\right)>0$ and $\lim _{i \rightarrow \infty} \mu_{i}=\infty, \mathcal{O}$ must be finite and $\mathcal{O}=\left\{i: i_{0} \leq i \leq i_{1}\right\}$.

(i) Assuming (S), we have $D\left(\mu_{0}\right)=D(0)=\left(b_{1} c_{2}-b_{2} c_{1}\right) u_{*} v_{*}<0$. From Theorem 4.4, with $k^{*}=m\left(\mu_{0}\right)+\sum_{i=i_{0}}^{i_{1}} m\left(\mu_{i}\right)$ being even, we have $i\left(T, \vec{u}_{*}\right)=1$. Therefore, combining with Proposition 4.3, we have

$$
\sum_{Z \cup\left\{\vec{u}_{*}\right\}} i\left(T, \vec{u}_{*}\right)=3
$$

Meanwhile $i(T, U)=1$, so there must be at least one more fixed point of $T$ in $U$ which is a nonconstant positive solution for the considered system.

(ii) For the "weak" competition case, $b_{1} / b_{2}>a_{1} / a_{2}>c_{1} / c_{2}$, we have $D\left(\mu_{0}\right)>0$. The proof is similar if one notes that $k^{*}=\sum_{i=i_{0}}^{i_{1}} m\left(\mu_{i}\right)$ is odd so that $\sum_{Z \cup\left\{\vec{u}_{*}\right\}} i\left(T, \vec{u}_{*}\right)=-1$.

Remark 4.8. If $D(\mu) \geq 0$ for all $\mu>0$, then Theorem 4.4 and (4.10) say that $i\left(T, \vec{u}_{*}\right)=1$ if $D\left(\mu_{0}\right)>0$ (or (W) holds) and $i\left(T, \vec{u}_{*}\right)=-1$ if $D\left(\mu_{0}\right)<0$ (or (S) holds). Comparing with Proposition 4.3, we see that $\sum_{Z \cup\left\{\vec{u}_{*}\right\}} i\left(T, \vec{u}_{*}\right)=1$, and that the above argument does not apply to give a conclusive answer.

The above theorem is stated in its most generality but its main assumption (4.12) is not easy to be verified in practice. However, if there exists an $i \geq 1$ such that $m\left(\mu_{i}\right)$ is odd, then one can take $k$ to be the smallest of such $i$ 's to have that $\sum_{i=1}^{k} m\left(\mu_{i}\right)$ is odd. The following special version of Theorem 4.7, when $i_{0}=1$ and $i_{1}=k$, could be more practical.

Corollary 4.9. Suppose that either (S) or (W) holds, and that there exists $k \geq 1$ such that $\sum_{i=1}^{k} m\left(\mu_{i}\right)$ is odd. If

$$
D\left(\mu_{1}\right)<0, \quad D\left(\mu_{k}\right)<0, \quad D\left(\mu_{k+1}\right)>0,
$$

then (1.1) with (4.1) has at least one positive nonconstant solution.

In the rest of this paper, we will explore several consequences of this result. Before doing so, we would like to point out that the analysis in this subsection has not used much of the structural condition (4.1) but the results in Section 2 and Section 3, which hold for much more general settings (see (YYY)). This generality would allow us to venture into other more general models.

However, for the sake of easy comparison with available literature, we will concentrate on (1.1) with (4.1). The analysis below also provides ways to study the effect of some parameters of the system on the existence of a nonconstant positive solution. Roughly speaking, no matter what competition situation ("strong" or "weak") is considered, the following theorem shows that suitably large (self or cross) diffusions will force the existence of such solutions. The main idea is to parameterize the diffusion matrix $\Theta$ by introducing a parameter $t$ in different 
coefficients of $P^{u}, P^{v}, Q^{u}, Q^{v}$, and then vary $t$ so that the condition (4.13) is fulfilled. One can see that this method can apply to many other interesting situations too. However, we restrain ourself to present only a few to illuminate the use of Corollary 4.9.

Theorem 4.10. Suppose that either (S) or (W) holds, and that there exists $k \geq 1$ such that $\sum_{i=1}^{k} m\left(\mu_{i}\right)$ is odd. We consider the following cases.

$$
\left\{\begin{array}{l}
\Theta=\left(\begin{array}{cc}
\delta_{1}+a_{11} u+t a_{12} v & t b_{11} u \\
b_{22} v & \delta_{2}+a_{21} u+a_{22} v
\end{array}\right), \\
a=a_{12} v_{*}\left(\delta_{2}+a_{21} u_{*}+a_{22} v_{*}\right)-b_{11} b_{22} u_{*} v_{*}, \\
b=\left(a_{12} c_{2} v_{*}-b_{11} b_{2} u_{*}\right) v_{*} .
\end{array}\right.
$$

$$
\left\{\begin{array}{l}
\Theta=\left(\begin{array}{cc}
\delta_{1}+a_{11} u+a_{12} v & b_{11} u \\
t b_{22} v & \delta_{2}+t a_{21} u+a_{22} v
\end{array}\right), \\
a=a_{21} u_{*}\left(\delta_{1}+a_{11} u_{*}+a_{12} v_{*}\right)-b_{11} b_{22} u_{*} v_{*}, \\
b=\left(b_{1} a_{21} u_{*}-b_{22} c_{1} v_{*}\right) u_{*} .
\end{array}\right.
$$

$$
\left\{\begin{array}{l}
\Theta=\left(\begin{array}{cc}
\delta_{1}+t a_{11} u+a_{12} v & t b_{11} u \\
b_{22} v & \delta_{2}+a_{21} u+a_{22} v
\end{array}\right), \\
a=a_{11} u_{*}\left(\delta_{2}+a_{21} u_{*}+a_{22} v_{*}\right)-b_{11} b_{22} u_{*} v_{*}, \\
b=\left(a_{11} c_{2}-b_{11} b_{2}\right) u_{*} v_{*} .
\end{array}\right.
$$

$$
\left\{\begin{array}{l}
\Theta=\left(\begin{array}{cc}
\delta_{1}+a_{11} u+a_{12} v & b_{11} u \\
t b_{22} v & \delta_{2}+a_{21} u+t a_{22} v
\end{array}\right), \\
a=a_{22} v_{*}\left(\delta_{1}+a_{11} u_{*}+a_{12} v_{*}\right)-b_{11} b_{22} u_{*} v_{*}, \\
b=\left(b_{1} a_{22}-b_{22} c_{1}\right) u_{*} v_{*} .
\end{array}\right.
$$

Suppose that $a_{12} a_{21}=b_{11} b_{22}$;

$$
\left\{\begin{array}{l}
\Theta=\left(\begin{array}{cc}
\delta_{1}+a_{11} u+t a_{12} v & t b_{11} u \\
t b_{22} v & \delta_{2}+t a_{21} u+a_{22} v
\end{array}\right), \\
a=a_{12} v_{*}\left(\delta_{2}+a_{22} v_{*}\right)+a_{21} u_{*}\left(\delta_{1}+a_{11} u_{*}\right), \\
b=b_{1} a_{21} u_{*}^{2}-\left(b_{2} b_{11}+c_{1} b_{22}\right) u_{*} v_{*}+c_{2} a_{12} v_{*}^{2} .
\end{array}\right.
$$

For each case, suppose that $a>0$ and $\mu_{k}<-b / a<\mu_{k+1}$; then there exists $t_{0}$ such that system (1.1) has at least one positive nonconstant solution for all $t>t_{0}$. 
Proof. We only prove the case (I) because the same argument applies to the other cases. From (4.11), a simple calculation gives

$$
D(\mu)=(a \mu+b) \mu t+\left(A \mu^{2}+B \mu+C\right),
$$

where $A, B, C$ are independent of $t$ and $a, b$ are defined as in the theorem.

Since $a>0$ and $0<\mu_{1} \leq \mu_{k}<-b / a$, we see that $\left(a \mu_{1}+b\right) \mu_{1}<0$ and $\left(a \mu_{k}+b\right) \mu_{k}<0$. Hence, from (4.14), for $t$ large enough we have $D\left(\mu_{1}\right)<0$ and $D\left(\mu_{k}\right)<0$.

Similarly, because $a>0$ and $\mu_{k+1}>-b / a$, we have $\left(a \mu_{k+1}+b\right) \mu_{k+1}>0$. Hence, $D\left(\mu_{k+1}\right)>0$ for $t$ sufficiently large.

Therefore, there exists $t_{0}>0$ such that (4.13) holds for $t>t_{0}$. Applying Corollary 4.9, we prove (I).

Several remarks should follow to discuss the conditions of this theorem as well as its biological interpretation.

Remark 4.11. From Definition (4.10) and Equation (4.14), it is easy to see that $\operatorname{det}(\Theta)=(a t+A)$. Hence, the condition $a>0$ is necessary here to guarantee the ellipticity condition of $(1.1)$ that requires $\operatorname{det}(\Theta)>0$ when $t$ is positively large.

Remark 4.12. The case $\left(\mathrm{I}^{\prime}\right)$ (respectively, $\left(\mathrm{II}^{\prime}\right)$ ) is just the counterpart of (I) (respectively, $\left(\mathrm{II}^{\prime}\right)$ ), where we interchange the roles of $u, v$. We only state them here for later references. These cases indicate that while diffusion and self diffusion tend to suppress pattern formation, cross-diffusion seems to help create patterns.

Remark 4.13. Case (I) implies that pattern formation can occur if one cross diffusion pressure is strong enough (while the other cross diffusion pressure stays fixed). On the other hand, since we allow $a_{12} \neq b_{11}$ we can refer to $a_{12}$, which goes with $v$, as the density cross diffusion; and $b_{11}$, which goes with $\nabla v$, as the gradient cross diffusion. Case (II) then concerns an interesting phenomenon where these two kinds of cross diffusions can be very different. We find that spatial segregation can also occur if the self diffusion and gradient cross diffusion pressures of one species are strong enough. Of course, one can try to introduce large $t$ only in front of the self diffusion $\delta_{1}$ or the density diffusion $a_{12}$ (or both). In these cases, however, $D\left(\mu_{i}\right)>0$ for all $i \geq 1$ when $t$ is large; and our method does not provide the desired coexistence (see Remark 4.8). It could be interesting to ask whether strong density cross diffusion alone would prevent pattern formation.

Remark 4.14. The case (III) positively answers a question in [19, page 83], where they consider $a_{12}=b_{11}$ and $a_{21}=b_{22}$, that "whether the nonconstant solution of the original SKT system exists when both cross-diffusion pressures are strong but qualitatively similar." Here, the ratios between the cross diffusion pressures are $b_{11} / b_{22}$ or $a_{12} / a_{21}$, which need not be very small or large. Note also that we only require $a_{12} a_{21}=b_{11} b_{22}$.

Next, we will see that some main coexistence results in [19] are just consequences of our above discussion. The following is a (more precise) restatement of 
[19, Theorem 1.3] concerning the "weak" competition case (W). Indeed, a careful inspection of the proof of [19, Theorem 1.3] reveals that the constants $C_{i}$ 's in the statement below should depend on the eigenvalues $\mu_{k}, \mu_{k+1}$.

Corollary 4.15. Suppose that $b_{11}=a_{12}, b_{22}=a_{21}$. Assume that (W) holds and there exists $k \geq 1$ such that $\sum_{i=1}^{k} m\left(\mu_{i}\right)$ is odd. Then the system (1.1) has at least one positive nonconstant solution in the following situations.

(i) $a_{1} / a_{2}>\frac{1}{2}\left(b_{1} / b_{2}+c_{1} / c_{2}\right)$ and $\delta_{2}+a_{22} v_{*} \in\left(C_{1}, C_{2}\right)$ for some positive constants $C_{1}<C_{2}$ depending on $a_{i}, b_{i}, c_{i}, \mu_{k}, \mu_{k+1}$. The cross diffusion $a_{12}=b_{11}$ is sufficiently large.

(ii) $a_{1} / a_{2}<\frac{1}{2}\left(b_{1} / b_{2}+c_{1} / c_{2}\right)$ and $\delta_{1}+a_{11} u_{*} \in\left(C_{3}, C_{4}\right)$ for some positive constants $C_{3}<C_{4}$ depending on $a_{i}, b_{i}, c_{i}, \mu_{k}, \mu_{k+1}$. The cross diffusion $a_{21}=b_{22}$ is sufficiently large.

Proof. We only give here the proof of the case (i). We consider the case (I) (case (I') for (ii)) of Theorem 4.10. Since $b_{11}=a_{12}, b_{22}=a_{21}$, we have $a=a_{12} v_{*}\left(\delta_{2}+a_{22} v_{*}\right), b=a_{12} v_{*}\left(c_{2} v_{*}-b_{2} u_{*}\right)$. As

$$
\left(u_{*}, v_{*}\right)=\left(\frac{a_{1} c_{2}-a_{2} c_{1}}{b_{1} c_{2}-b_{2} c_{1}}, \frac{b_{1} a_{2}-b_{2} a_{1}}{b_{1} c_{2}-b_{2} c_{1}}\right),
$$

we have

$$
\begin{aligned}
\frac{-b}{a}=\frac{b_{2} u_{*}-c_{2} v_{*}}{\delta+a_{22} u_{*}}=\frac{C_{*}}{\delta+a_{22} u_{*}}, \\
\text { where } C_{*}=\frac{2 a_{2} b_{2} c_{2}\left[a_{1} / a_{2}-\frac{1}{2}\left(b_{1} / b_{2}+c_{1} / c_{2}\right)\right]}{b_{1} c_{2}-b_{2} c_{1}}>0 .
\end{aligned}
$$

Let $C_{1}=C_{*} / \mu_{k+1}, C_{2}=C_{*} / \mu_{k}$. When $\delta+a_{22} v_{*} \in\left(C_{1}, C_{2}\right)$, we have $\mu_{k}<$ $-b / a<\mu_{k+1}$. Applying Theorem 4.10, with $t$ being large, we complete the proof.

For the "strong" competition case, the following strengthens [19, Theorem 1.4] in the sense that the intrinsic self diffusions $\delta_{i}$ do not have to be large. We will not present the proof here since it is similar to the above proof.

Corollary 4.16. Suppose that $b_{11}=a_{12}, b_{22}=a_{21}$. Assume that (S) holds and there exists $k \geq 1$ such that $\sum_{i=1}^{k} m\left(\mu_{i}\right)$ is odd. Then the system (1.1) has at least one positive nonconstant solution in the following situations.

(i) $a_{1} / a_{2}<\frac{1}{2}\left(b_{1} / b_{2}+c_{1} / c_{2}\right)$ and $\delta_{2}+a_{22} v_{*} \in\left(C_{5}, C_{6}\right)$ for some positive constants $C_{5}<C_{6}$ depending on $a_{i}, b_{i}, c_{i}, \mu_{k}, \mu_{k+1}$. The cross diffusion $a_{12}=b_{11}$ is sufficiently large.

(ii) $a_{1} / a_{2}>\frac{1}{2}\left(b_{1} / b_{2}+c_{1} / c_{2}\right)$ and $\delta_{1}+a_{11} u_{*} \in\left(C_{7}, C_{8}\right)$ for some positive constants $C_{7}<C_{8}$ depending on $a_{i}, b_{i}, c_{i}, \mu_{k}, \mu_{k+1}$. The cross diffusion $a_{21}=b_{22}$ is sufficiently large. 
Remark 4.17. The results in this sub-section continue to hold if a much more general structural condition on the diffusion matrix like $\left(Y Y Y^{\prime}\right)$ is in place. One would easily see that Theorem 4.10 is the only place where we consider the structure condition (4.1). In addition, we could only require that the partial derivatives of $\tilde{f}, \tilde{g}(f=u \tilde{f}, g=v \tilde{g})$ are negative.

\section{OTHER MOdELS}

We conclude our paper by some examples concerning some other applications of our previous results. In particular, we will assume the structure $\left(Y^{\prime} Y^{\prime}\right)$ on the diffusion matrix $\Theta$ whose entries can be negative. Obviously, such a generalization would open a wealth of interesting investigations on the effect of different kinds of nonlinear dispersive forces in the spatial segregation question. The uniform bounds in Section 2, the fixed point index results in Section 3, and the Theorem 4.4 are the key ingredients for such explorations.

For the sake of brevity, we will restrict ourself to the Neumann boundary conditions and the following structure on the reaction terms.

(F) Assume that $f(u, v)=u \tilde{f}(u, v)$ and $g(u, v)=v \tilde{g}(u, v)$. Moreover,

$$
\tilde{f}(u, v)=f^{u}(u)+f^{v}(v), \quad \tilde{g}(u, v)=g^{u}(u)+g^{v}(v),
$$

for some functions $f^{u}, f^{v}, g^{u}, g^{v}$ that satisfy

$$
\begin{aligned}
& f^{u}(0), g^{v}(0)>0, \quad f^{v}(0)=g^{u}(0)=0, \\
& \text { and } \quad \lim _{u \rightarrow \infty} f^{u}(u)=\lim _{v \rightarrow \infty} g^{v}(v)=-\infty .
\end{aligned}
$$

There is a unique positive solution $\left(u_{*}, v_{*}\right)$ to

$$
f^{u}(u)+f^{v}(v)=0, \quad g^{u}(u)+g^{v}(v)=0 .
$$

Here, $f^{u}, g^{v}$ are respectively the birth-death rates of $u, v$, whereas $f^{v}, g^{u}$ describe the reaction between the species. The assumption (5.1) is very common in the context of biology.

Define the operator $T$ as in Section 3. Following the calculation in Proposition 4.3 (see (4.8)) and using the facts that $f^{u}(0), g^{v}(0)>0, f^{v}(0)=g^{u}(0)=$ 0 , we easily see that $(\mathrm{E})$ holds and $i(T, 0)=0$.

Clearly, the solution $\left(u_{*}, v_{*}\right)$ of (5.2) is the positive constant solution to (1.1). We then compute the fixed point index of $T$ at $\left(u_{*}, v_{*}\right)$. It is easy to see that Theorem 4.4 in Section 4.2 is still valid. Following the calculation in that 
section we see that

$$
\begin{aligned}
& \mathcal{N}_{i}=\frac{1}{d\left(\vec{u}_{*}\right) \mu_{i}+k}\left(\begin{array}{cc}
Q^{v} & -P^{v} \\
-Q^{u} & P^{u}
\end{array}\right) \\
& \times\left[-\left(\begin{array}{ll}
\frac{\partial \tilde{f}}{\partial u}\left(u_{*}, v_{*}\right) u_{*} & \frac{\partial \tilde{f}}{\partial v}\left(u_{*}, v_{*}\right) u_{*} \\
\frac{\partial \tilde{g}}{\partial u}\left(u_{*}, v_{*}\right) v_{*} & \frac{\partial \tilde{g}}{\partial v}\left(u_{*}, v_{*}\right) v_{*}
\end{array}\right)+\mu_{i}\left(\begin{array}{ll}
P^{u} & P^{v} \\
Q^{u} & Q^{v}
\end{array}\right)\right] .
\end{aligned}
$$

and (see (4.10))

$$
D\left(\mu_{i}\right):=\operatorname{det}\left[\left(\begin{array}{ll}
b_{1} u_{*} & c_{1} u_{*} \\
b_{2} v_{*} & c_{2} v_{*}
\end{array}\right)+\mu_{i}\left(\begin{array}{ll}
P^{u} & P^{v} \\
Q^{u} & Q^{v}
\end{array}\right)\right]
$$

with

$$
\begin{array}{ll}
b_{1}=-\frac{\partial \tilde{f}}{\partial u}\left(u_{*}, v_{*}\right), & c_{1}=-\frac{\partial \tilde{f}}{\partial v}\left(u_{*}, v_{*}\right), \\
b_{2}=-\frac{\partial \tilde{g}}{\partial u}\left(u_{*}, v_{*}\right), & c_{2}=-\frac{\partial \tilde{g}}{\partial v}\left(u_{*}, v_{*}\right) .
\end{array}
$$

From Theorem 4.4, we then have

$$
i\left(T, \vec{u}_{*}\right)=(-1)^{k^{*}}, \quad \text { with } k^{*}=\sum_{\operatorname{det} D\left(\mu_{i}\right)<0} m\left(\mu_{i}\right) .
$$

From (5.1) the equations $f^{u}(u)=0$ and $g^{v}(v)=0$ have positive roots which are the semitrivial solutions on the $u, v$ axes. In the sequel, we will study the fixed point indices of $T$ at these solutions and derive sufficient conditions for the existence of nonconstant solutions.

5.1. The prey-predator case We consider the case where the prey $u$ is deprived by and tries to avoid the predator $v$, whereas $v$ benefits from $u$ and moves toward high concentration areas of $u$. Mathematically, this can be modeled by assuming that

$$
P^{v}(u, v)>0, Q^{u}(u, v)<0, f^{v}(v)<0, g^{u}(u)>0, \quad \forall u, v>0 .
$$

For $k$ large, we easily see that $k P^{u}+Q^{u}>0$. Thus, (A.2) is verified. For (A.3), we need to assume that $k u \tilde{f}(u, v)+v \tilde{g}(u, v)$ is negative when either $u$ or $v$ is large. Thanks to $(\mathrm{F})$ this means

$$
k u\left(f^{u}(u)+f^{v}(v)\right)+v\left(g^{u}(u)+g^{v}(v)\right)<0, \quad \text { when either } u \text { or } v \text { is large. }
$$


This condition will be satisfied if the functions $f^{u}, f^{v}, g^{u}, g^{v}$ are polynomials of the same order. This can be seen by choosing $k$ sufficiently large and a simple use of Young's inequality.

We then study the semitrivial solutions, which can be found by solving $f^{u}(u)=$ 0 or $g^{v}(v)=0$. Let $u>0$ be a root of $f^{u}(u)=0$. Proceed as in Proposition 4.3 and consider the eigenvalue problem

$$
-d(u, 0) \Delta \varphi+k \varphi=\lambda^{-1} \frac{\partial}{\partial v} G(u, 0) \varphi .
$$

We see that $\varphi=1$ is an eigenvector corresponding to the eigenvalue

$$
\lambda=\frac{P^{u}(u, 0) \tilde{g}(u, 0)+k}{k}=\frac{P^{u}(u, 0)\left(g^{u}(u)+g^{v}(0)\right)+k}{k},
$$

which is greater than 1 because $g^{u}(u)+g^{v}(0)>0$, thanks to (5.5). Thus, $\left(D_{-}\right)$ holds and $i\left(T, Z_{1}\right)=0$.

On the other hand, if $v>0$ is a root of $g^{v}(v)=0$, then we need to look at the eigenvalue problem

$$
-d(u, 0) \Delta \varphi+k \varphi=\lambda^{-1} \frac{\partial}{\partial u} F(0, v) \varphi,
$$

whose principal eigenvalue is

$$
\lambda=\frac{Q^{v}(0, v) \tilde{f}(0, v)+k}{k}=\frac{Q^{v}(0, v)\left(f^{v}(v)+f^{u}(0)\right)+k}{k} .
$$

Thus, $\lambda>1$ (respectively, $\lambda<1$ ) if $f^{v}(v)+f^{u}(0)$ is positive (respectively, negative).

The above calculation leads to the following result.

Theorem 5.1. Assume (F) and (5.5). The system (1.1) has a positive nonconstant solution if for all positive root $v$ of $g^{v}(v)=0$ we have either

(i) $f^{v}(v)+f^{u}(0)<0$, or

(ii) $f^{v}(v)+f^{u}(0)>0$ and $k^{*}$ in (5.4) is odd.

Proof. For (i), the above calculation shows that $i\left(T, Z_{2}\right)=1$ and $i\left(T, Z_{1}\right)=$ 0 . Since $i(T, U)=1$ and $i\left(T, \vec{u}_{*}\right)= \pm 1, \sum_{\vec{u} \in Z \cup\left\{\vec{u}_{*}\right\}} i(T, \vec{u}) \neq i(T, U)$.

In case (ii), we now have $i\left(T, Z_{2}\right)=0$ and $i\left(T, \vec{u}_{*}\right)=-1$ (because $k^{*}$ is odd). Thus, $\sum_{\vec{u} \in Z \cup\left\{\vec{u}_{*}\right\}} i(T, \vec{u}) \neq i(T, U)$.

In both cases, from the property of fixed point index, the nonconstant coexistence follows. 
5.2. The Cooperative case We now consider the case when $u, v$ mutually benefit each other and strive to move toward the other's high concentration areas. The mathematical assumptions then read

$$
P^{v}(u, v), Q^{u}(u, v)<0, \quad f^{v}(v), g^{u}(u)>0, \quad \forall u, v>0 .
$$

To justify (A.2), we need to find $k$ such that $k P^{u}+Q^{u}>0$ and $k P^{v}+Q^{v}>0$. In general, it is not easy to find such $k$. However, for diffusion matrices of the form (4.1) (with $b_{11}, b_{22}<0$ ) we can see that any $k$ that satisfies $-b_{11} / a_{21}>k>$ $-b_{22} / a_{12}$ would serve our purpose.

For (A.3), we need to assume that

$$
k u\left(f^{u}(u)+f^{v}(v)\right)+v\left(g^{u}(u)+g^{v}(v)\right)<0, \quad \text { when either } u \text { or } v \text { is large. }
$$

Again, from Young's inequality, this condition will be satisfied if the functions $f^{u}, f^{v}, g^{u}, g^{v}$ are polynomials of the same order and with suitable coefficients. Notice that we can no longer choose $k$ arbitrarily large in this case.

Concerning the semitrivial solutions, for $u>0$ being a root of $f^{u}(u)=0$, we also consider the eigenvalue problem

$$
-d(u, 0) \Delta \varphi+k \varphi=\lambda^{-1} \frac{\partial}{\partial v} G(u, 0) \varphi .
$$

The principal eigenvalue

$$
\lambda=\frac{P^{u}(u, 0) \tilde{g}(u, 0)+k}{k}=\frac{P^{u}(u, 0)\left(g^{u}(u)+g^{v}(0)\right)+k}{k},
$$

which is now greater than 1 because $g^{u}(u)+g^{v}(0)>0$, thanks to (5.6). Thus, $\left(D_{-}\right)$holds and $i\left(T, Z_{1}\right)=0$. The same argument applies to the semitrivial solution $(0, v)$, with $v>0$ being a root of $g^{v}(v)=0$. Thus, $\sum_{\vec{u} \in Z} i(T, \vec{u})=0$. To guarantee the nonconstant coexistence, we need $i\left(T, \vec{u}_{*}\right)=-1$ or $k^{*}$ to be odd.

We leave the statement of the theorems to interested readers but note that sufficient conditions for the oddness of $k^{*}$ can be derived in a similar manner as in Theorem 4.10. We should also point out that it is also possible to have more than one positive solution to (5.2) and the analysis can be done at each of these solutions to obtain several other sufficient conditions for pattern formation.

Acknowledgement The first author is partially supported by NSF Grant \#DMS0305219, Applied Mathematic Program.

\section{REFERENCES}

[1] H. AMANn, Fixed point equations and nonlinear eigenvalue problems in ordered Banach spaces, SIAM Rev. 18 (1976), 620-709, http://dx.doi.org/10.1137/1018114. MR 0415432 (54 \#3519) 
[2] L. C. Evans, Partial Differential Equations, Graduate Studies in Mathematics, vol. 19, American Mathematical Society, Providence, RI, 1998, ISBN 0-8218-0772-2. MR 1625845 (99e:35001)

[3] R. S. CANTREll and C. COSNER, Diffusive logistic equations with indefinite weights: population models in disrupted environments. II, SIAM J. Math. Anal. 22 (1991), 1043-1064, http://dx.doi.org/10.1137/0522068. MR 1112065 (92h:92015)

[4] W. CHEN and R. PENG, Stationary patterns created by cross-diffusion for the competitor-competitor-mutualist model, J. Math. Anal. Appl. 291 (2004), 550-564, http://dx.doi.org/10.1016/j.jmaa.2003.11.015. MR 2039069 (2005a:35156)

[5] X. CHEN, Y. QI, and M. WANG, Steady states of a strongly coupled prey-predator model, Discrete Contin. Dyn. Syst. (2005), 173-180. MR 2192672 (2006h:35057)

[6] G. CUPINI and R. PETTI, Morrey spaces and local regularity of minimizers of variational integrals, Rend. Mat. Appl. (7) 21 (2001), 121-141. MR 1884939 (2002m:49055) (English, with English and Italian summaries)

[7] E. N. DANCER and Y. H. DU, Positive solutions for a three-species competition system with diffusion. I. General existence results, Nonlinear Anal. 24 (1995), 337-357, http://dx.doi.org/10.1016/0362-546X(94)E0063-M. MR 1312772 (96c:92028)

[8] M. GIAQUinTA, Multiple Integrals in the Calculus of Variations and Nonlinear Elliptic Systems, Annals of Mathematics Studies, vol. 105, Princeton University Press, Princeton, NJ, 1983, ISBN 0-691-08330-4, 0-691-08331-2. MR 717034 (86b:49003)

[9] D. Gilbarg and N. S. Trudinger, Elliptic Partial Differential Equations of Second Order, 2nd ed., Grundlehren der Mathematischen Wissenschaften [Fundamental Principles of Mathematical Sciences], vol. 224, Springer-Verlag, Berlin, 1983, ISBN 3-540-13025-X. MR 737190 (86c:35035)

[10] K. KUTO, Stability of steady-state solutions to a prey-predator system with cross-diffusion, J. Differential Equations 197 (2004), 293-314, http://dx.doi.org/10.1016/j.jde.2003.10.016. MR 2034162 (2004k:35174)

[11] K. KUTO and Y. YAMADA, Multiple existence and stability of steady-states for a prey-predator system with cross-diffusion, Nonlocal Elliptic and Parabolic Problems, Banach Center Publ., vol. 66, Polish Acad. Sci., Warsaw, 2004, pp. 199-210. MR 2143365 (2006a:35137)

[12] _. Multiple coexistence states for a prey-predator system with cross-diffusion, J. Differential Equations 197 (2004), 315-348, http://dx.doi.org/10.1016/j.jde.2003.08.003. MR 2034163 (2004m:35070)

[13] O. A. LAdyzhensKaya and N. N. URAL'TSEVA, Linear and Quasilinear Elliptic Equations, Translated from the Russian by Scripta Technica, Inc. Translation editor: Leon Ehrenpreis, Academic Press, New York, 1968. MR 0244627 (39 \#5941)

[14] O. A. Ladyzhenskaya, V. A. SOlOnNiKOV, and N. N. URAL'CEVA, Linear and Quasilinear Equations of Parabolic Type, AMS Transl. Monographs, 1968. MR 0241821 (39 \#3159a)

[15] D. LE, Coexistence with chemotaxis, SIAM J. Math. Anal. 32 (2000), 504-521 (electronic). MR 1786154 (2001i:35074)

[16] - Global existence for a class of strongly coupled parabolic systems, Ann. Mat. Pura Appl. (4) 185 (2006), 133-154, http://dx.doi.org/10.1007/s10231-004-0131-7. MR 2179585 (2006i:35190)

[17] D. LE and T. T. NGUYEN, Everywhere regularity of solutions to a class of strongly coupled degenerate parabolic systems, Comm. Partial Differential Equations 31 (2006), 307-324, http://dx.doi.org/10.1080/0360530050036038. MR 2209756 (2007b:35161) 
[18] _ Persistence for a class of triangular cross diffusion parabolic systems, Adv. Nonlinear Stud. 5 (2005), 493-514. MR 2180580 (2006e:35199)

[19] Y. LOU and W.-M. NI, Diffusion, self-diffusion and cross-diffusion, J. Differential Equations 131 (1996), 79-131, http://dx.doi.org/10.1006/jdeq.1996.0157. MR 1415047 (97i:35086)

[20] H. Matano and M. Mimura, Pattern formation in competition-diffusion systems in nonconvex domains, Publ. Res. Inst. Math. Sci. 19 (1983), 1049-1079. MR 723460 (85e:35065)

[21] W.-M. NI, Diffusion and cross-diffusion in pattern formation, Atti Accad. Naz. Lincei Cl. Sci. Fis. Mat. Natur. Rend. Lincei (9) Mat. Appl. 15 (2004), 197-214. MR 2148879 (2006c:35092)

[22] D. K. Palagachev, M. A. Ragusa, and L. G. Softova, Regular oblique derivative problem in Morrey spaces, Electron. J. Differential Equations (2000), No. 39, 17 pp. (electronic). MR 1764709 (2001d:35043)

[23] C. V. PAO, Strongly coupled elliptic systems and applications to Lotka-Volterra models with crossdiffusion, Nonlinear Anal. 60 (2005), 1197-1217, http://dx.doi.org/10.1016/j.na.2004.10.008. MR 2112950 (2005i:35072)

[24] W. H. RUAN, Positive steady-state solutions of a competing reaction-diffusion system with large cross-diffusion coefficients, J. Math. Anal. Appl. 197 (1996), 558-578, http://dx.doi.org/10.1006/jmaa.1996.0039. MR 1372198 (97d:92014)

[25] _ A competing reaction-diffusion system with small cross-diffusions, Canad. Appl. Math. Quart. 7 (1999), 69-91. MR 1687465 (2000h:92030)

[26] K. RYU and I. AHN, Coexistence theorem of steady states for nonlinear self-cross diffusion systems with competitive dynamics, J. Math. Anal. Appl. 283 (2003), 46-65, http://dx.doi.org/10.1016/S0022-247X(03)00162-8. MR 1994171 (2004h:35054)

[27] N. Shigesada, K. KaWASAKI, and E. Teramoto, Spatial segregation of interacting species, J. Theoret. Biol. 79 (1979), 83-99, http://dx.doi.org/10.1016/0022-5193(79)90258-3. MR 540951 (80e:92038)

[28] Y. WU, The instability of spiky steady states for a competing species model with cross diffusion, J. Differential Equations 213 (2005), 289-340, http://dx.doi.org/10.1016/j.jde.2004.08.015. MR 2142368 (2006a:35139)

[29] Y. YAMADA, Coexistence states for Lotka-Volterra systems with cross-diffusion, Proc. Operator Theory and Its Applications, Winnipeg, MB, 1998, Fields Inst. Commun., vol. 25, Amer. Math. Soc., Providence, RI, 2000, pp. 551-564. MR 1759568 (2001g:35085)

[30] Eberhard Zeidler, Nonlinear Functional Analysis and Its Applications. I, Springer-Verlag, New York, 1986, ISBN 0-387-90914-1, Fixed-point theorems; Translated from the German by Peter R. Wadsack. MR 816732 (87f:47083)

DUNG LE:

Department of Applied Mathematics

University of Texas at San Antonio

6900 North Loop 1604 West

San Antonio, TX 78249, U.S.A.

E-MAIL: dle@math.utsa.edu

LINH V. NGUYEN:

Department of Applied Mathematics University of Texas at San Antonio 6900 North Loop 1604 West

San Antonio, TX 78249, U.S.A. 
CURRENT ADDRESS:

Department of Mathematics

Mailstop 3368

Texas A \& M University

College Station, TX 77843-3368, U.S.A.

E-MAIL: lnguyen@math.tamu.edu

ToAn T. NGuyen: Department of Applied Mathematics

University of Texas at San Antonio

6900 North Loop 1604 West

San Antonio, TX 78249, U.S.A.

CURRENT ADDRESS: Department of Mathematics

Indiana University

831 East 3rd St.

Bloomington, IN 47405, U.S.A.

E-MAIL: nguyentt@indiana.edu

KEY WORDS AND PHRASES:

strongly coupled elliptic systems; regularity; fixed point index; existence.

2000 Mathematics Subject Classification: 35J55; 35J60; 35B65; 35B45.

Received: February 28th, 2006.

Article electronically published on May 4th, 2007. 